Check for updates

Cite this: Chem. Sci., 2020, 11, 812

๑ All publication charges for this article have been paid for by the Royal Society of Chemistry

Received 11th September 2019 Accepted 24th November 2019

DOI: $10.1039 / c 9 s c 04587 b$

rsc.li/chemical-science

\section{Controlling the structure and photophysics of fluorophore dimers using multiple cucurbit[8]uril clampings $\dagger$}

\author{
Guanglu Wu, (D) a Youn Jue Bae, ${ }^{b}$ Magdalena Olesińska, ${ }^{a}$ Daniel Antón-García, \\ István Szabó, ${ }^{d}$ Edina Rosta, ${ }^{d}$ Michael R. Wasielewski (iD ${ }^{b}$ and Oren A. Scherman (iD *a
}

\begin{abstract}
A modular strategy has been employed to develop a new class of fluorescent molecules, which generates discrete, dimeric stacked fluorophores upon complexation with multiple cucurbit[8]uril macrocycles. The multiple constraints result in a "static" complex (remaining as a single entity for more than $30 \mathrm{~ms}$ ) and facilitate fluorophore coupling in the ground state, showing a significant bathochromic shift in absorption and emission. This modular design is surprisingly applicable and flexible and has been validated through an investigation of nine different fluorophore cores ranging in size, shape, and geometric variation of their clamping modules. All fluorescent dimers evaluated can be photo-excited to atypical excimer-like states with elongated excited lifetimes (up to $37 \mathrm{~ns}$ ) and substantially high quantum yields (up to 1). This strategy offers a straightforward preparation of discrete fluorophore dimers, providing promising model systems with explicitly stable dimeric structures and tunable photophysical features, which can be utilized to study various intermolecular processes.
\end{abstract}

\section{Introduction}

Coupling two fluorophores within a sufficiently short distance for an extended period of time is crucial for both theoretical and experimental investigation of intermolecular processes such as charge transfer, ${ }^{1}$ excimer formation, ${ }^{2,3}$ long- or short-range exciton coupling, ${ }^{4,5}$ and singlet fission..$^{6-8}$ Stacking together precisely two fluorophores in an aqueous solution, however, remains a substantial challenge as most aromatic hydrocarbons show a tendency to aggregate unpredictably (forming clusters of arbitrary numbers of molecules). ${ }^{9-11}$ To prevent fluorophores from aggregation in aqueous solution, a supramolecular approach has been established to "mechanically" separate fluorescent molecules through encapsulation by macrocycles. ${ }^{12-16}$ A popular class of macrocyclic hosts utilized for this purpose is cucurbit[ $n]$ uril $(\mathrm{CB}[n], n=5-8,10)$, which contains

\footnotetext{
${ }^{a}$ Melville Laboratory for Polymer Synthesis, Department of Chemistry, University of Cambridge, Lensfield Road, Cambridge, CB2 1EW, UK. E-mail: oas23@cam.ac.uk

${ }^{b}$ Department of Chemistry, Institute for Sustainability and Energy at Northwestern, Northwestern University, Evanston, Illinois 60208-3113, USA

'Department of Chemistry, University of Cambridge, Lensfield Road, Cambridge, CB2 $1 E W, U K$

${ }^{d}$ Department of Chemistry, King's College London, 7 Trinity Street, London, SE1 1DB, UK

$\uparrow$ Electronic supplementary information (ESI) available: Materials and methods, synthesis and characterization, DOSY results, complexation studies of nine fluorescent molecules, VT-NMR, transient absorption study, and MD simulation of the Ant910Me $\mathrm{M}_{2}-\mathrm{CB}[8]_{2}$ complex in a water box: MD_Ant910Me_CB[8].avi. See DOI: $10.1039 / \mathrm{c} 9 \mathrm{sc} 04587 \mathrm{~b}$
}

a cavity that enables the inclusion of various guest molecules and exhibits particularly high affinity towards positivelycharged species. ${ }^{17,18}$ As an example, $\mathrm{CB}[7]$ is a promising host for the complexation of various fluorescent dyes, ${ }^{19}$ resulting in significant changes in photophysical properties such as antiphotobleaching ${ }^{20}$ and emission enhancement. ${ }^{21-23}$ This is attributed to the hydrophobic environment provided by the $\mathrm{CB}$ cavity as well as mechanical protection by the macrocycle against aggregation and quenching. ${ }^{12}$

Dimeric fluorophore stacking, however, is unlikely to be realized by $\mathrm{CB}[7]-m e d i a t e d$ complexation as its relatively small cavity only allows the complexation with one single guest molecule or, more strictly speaking, one binding moiety on a guest molecule. On the other hand, $\mathrm{CB}[8]$, a larger cucurbituril homologue, is capable of simultaneously encapsulating two guest moieties yielding either a heteroternary ${ }^{24}$ or homoternary complex. ${ }^{25}$ Although $\mathrm{CB}[8]$-mediated ternary complexation may achieve stacking of two fluorophores, several limitations exist as the fluorophores are required to have the right shape, size and charge distribution to undergo complexation with $\mathrm{CB}[8] .^{26}$ Moreover, they must align along the principal symmetry axis of the CB cavity limiting the way in which they stack. ${ }^{27}$ In case of a stepwise complexation of two guests with $\mathrm{CB}[8]$, formation of a dynamic ternary complex is evident by the significant signal broadening in NMR spectra. ${ }^{24,25}$ This dynamic complex results in a short-lived coupling between the two stacked fluorophores that is insufficient to allow for the investigation of specific intermolecular processes. 
Recently, we have found that the dynamic exchange kinetics between the guests and $\mathrm{CB}[8]$ hosts are dramatically reduced through the formation of $2: 2$ quaternary complexes, ${ }^{28}$ in which two elongated guests such as diarylviologen derivatives are "clamped" in place by two $\mathrm{CB}[8]$ hosts into a multicomponent complex. The simultaneous formation of two ternary motifs within a discrete complex decreases the likelihood of dissociation compared to a typical ternary complex. ${ }^{28}$ The formation of $2: 2$ complexes opposed to elongated supramolecular polymers requires a small change in the conformational entropy during complexation, i.e. a molecule with significant rigidity. ${ }^{28,29}$ For instance, various rigid molecular moieties such as benzidine, ${ }^{30}$ benzothiazole, ${ }^{31,32}$ arylpyridinium, ${ }^{28,33}$ arylterpyridyl, ${ }^{34}$ bipyridinium, ${ }^{35}$ and benzimidazole ${ }^{29}$ have been employed to produce $\mathrm{CB}[8]$-mediated $2: 2$ complexes. Herein, we present a general and modular strategy towards the dimerization of arbitrary functional components (fluorophores in this work) by connecting them to multiple rigid modules that can be "clamped" together by $\mathrm{CB}[8]$ complexation. We use arylpyridinium moieties as the rigid "clamping" module (Fig. 1a) and exploit the modular strategy for designing fluorescent complexes in water comprised of two fluorophores that are stacked in a specific configuration with a constraint applied by $\mathrm{CB}[8]$ macrocycles at multiple points.

As illustrated in Fig. 1a, water-soluble fluorescent molecules are designed to incorporate a fluorescent core between two positively charged clamping modules, which in this work are arylpyridinium motifs originating from previously studied diarylviologen derivatives. ${ }^{28}$ When one equivalent (equiv.) of $\mathrm{CB}[8]$ is added to the system with one equiv. of guest molecule, two clamping modules are expected to bring together two guest molecules yielding a $2: 2$ quaternary complex. The fluorophore cores from each guest molecule are brought to close proximity to each other as a consequence of the assembly, resulting in preorganized dimeric fluorophore stacking. The preorganized dimer complex is stabilized by multiple $\mathrm{CB}[8]$ clamps, which ensures interaction between fluorophores for a sufficiently long period of time, endowing the complex with emergent photophysical properties. As the fluorophore modules are not encapsulated by $\mathrm{CB}[8]$ (Fig. 1c), a variety of fluorophores, including those with sizes substantially larger than the $\mathrm{CB}[8]$ cavity, can be employed as functional cores in this modular strategy (Scheme S1 $\dagger$ ). Moreover, the photophysical properties of the resultant complexes can be readily customized through altering fluorophores as well as the clamping modules. As exemplified in Fig. 1b, dimeric stacking still occurs even when the two clamping modules are non-parallel to each other (separated by an angle $<180^{\circ}$ ). The flexibility offered by this modular design provides a molecular toolbox and platform in which a wide range of fluorophores can be readily studied in their discrete monomeric or dimeric states facilitating future investigations of quantum optical phenomena.

\section{Results and discussion}

Fluorescent molecules are designed by bridging two arylpyridinium motifs with a central fluorophore core. Nine phenyl, a)
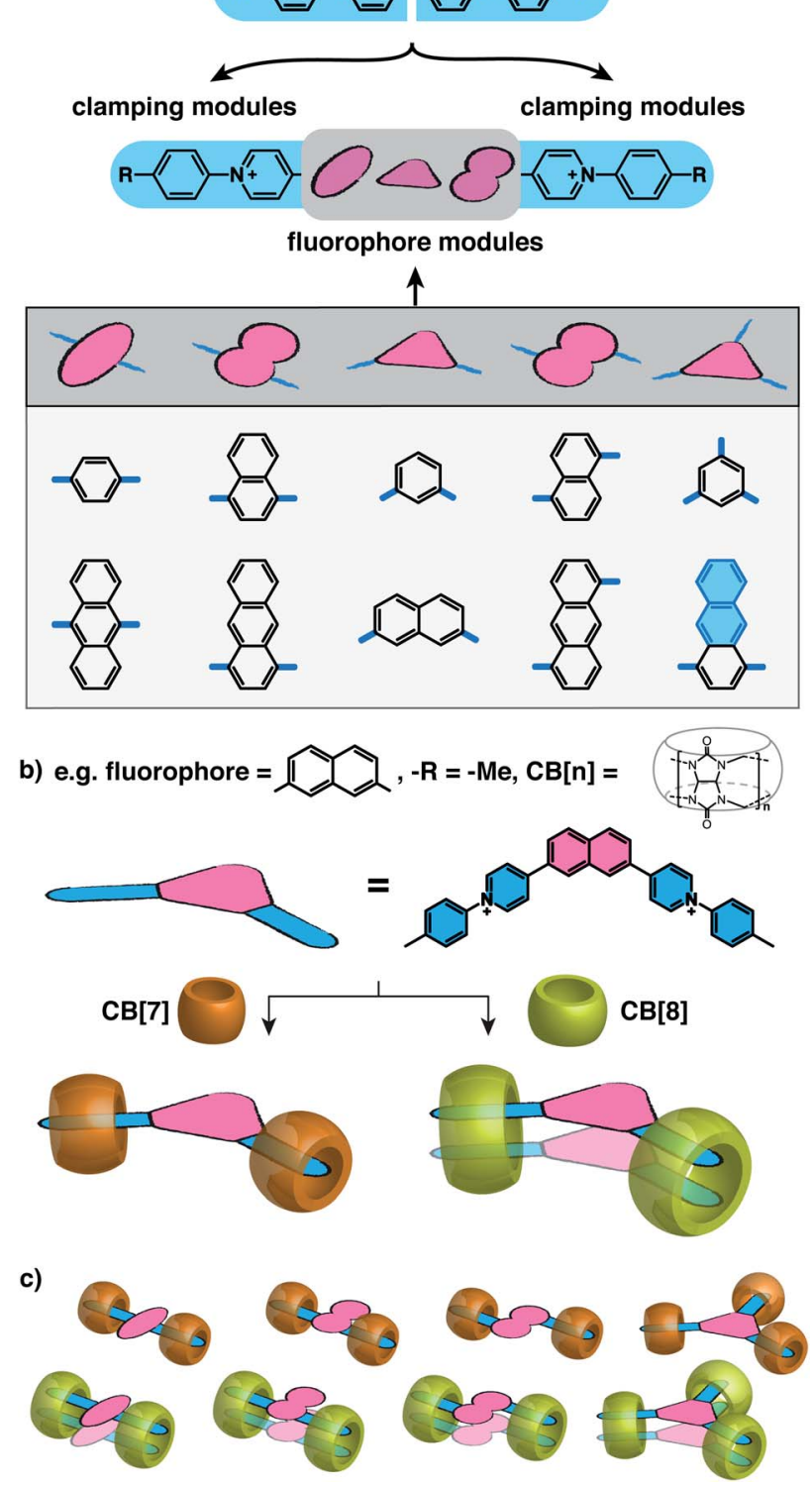

Fig. 1 Modular strategy for designing fluorescent molecules (a) by plugging in a fluorophore module between two positively charged clamping modules, which herein are arylpyridinium moieties originated from diarylviologen derivatives. Following this strategy, (b) 2,7naphthalene with non-parallel clamping modules, as an example, or (c) other fluorophores with various arrangement of clamping modules are expected to form a preorganized dimer constrained by CB[8]-mediated multiple clamping or a monomeric state of fluorophores protected by $\mathrm{CB}$ [7] from aggregation. The $\mathrm{Cl}^{-}$counterions are omitted for clarity.

naphthyl, or anthracenyl homologues are investigated as the fluorophore cores in this study (Fig. 1 and Scheme S1†). The general synthesis (Scheme S2 $\dagger$ ) of the molecules starts with Suzuki-Miyaura cross-coupling ${ }^{36}$ of two pyridin-4-yl groups onto the fluorophore core, followed by transformation of the pyridin4-yl groups into arylpyridinium salts through a Zincke reaction. ${ }^{37-39}$ A complete study was carried out on Ant910Me, which contains a 9,10-anthracenyl ("Ant910") as the central core and $p$ - 
tolyl pyridiniums ("Me") as clamping modules (Fig. 1a), which is presented here as a typical case prior to a further general discussion.

Guest molecule (G) Ant910Me is found to form 1:2 complexes with $\mathrm{CB}[7]$, denoted $\mathrm{G}_{1}-\mathrm{CB}[7]_{2}$, and $2: 2$ complexes with $\mathrm{CB}[8]$, denoted $\mathrm{G}_{2}-\mathrm{CB}[8]_{2}$. The complex formations are verified by several NMR techniques including ${ }^{1} \mathrm{H}$ NMR, 2D nuclear Overhauser spectroscopy (NOESY), and diffusion ordered spectroscopy (DOSY).

\section{$2.1 \mathrm{G}_{1}-\mathrm{CB}[7]_{2}$ : a discrete monomeric state}

The way in which $\mathrm{CB}[n]$ binds to guest molecules can be precisely probed by ${ }^{1} \mathrm{H}$ NMR. Protons residing inside the $\mathrm{CB}$ cavity typically exhibit upfield chemical shifts of $c a .1 \mathrm{ppm}$; while protons located outside and proximate to the CB portals will display downfield shifts. ${ }^{40}{ }^{1} \mathrm{H}$ NMR spectra of Ant910Me (Fig. 2a) and its $\mathrm{CB}$ [7] complex (Fig. 2b) demonstrate significant upfield shift for the $\mathrm{H}^{\mathrm{c}, \mathrm{d}, \mathrm{e}, \mathrm{f}}$ upon complexation, which indicates that the entire tolyl moiety resides inside the $\mathrm{CB}[7]$ cavity along with a part of the pyridinium group. Meanwhile, a slight downfield shift of $\mathrm{H}^{\mathrm{g}, \mathrm{h}}$ confirms that the anthracenyl core is located outside the $\mathrm{CB}[7]$ portals.

CB proton signals ranging from 4 to $6 \mathrm{ppm}$ split into two sets of equivalent doublets (Fig. 2b). The signal splitting suggests that the rate of $\mathrm{CB}$ [7] flipping around the tolyl moieties falls in the slow exchange limit with respect to the NMR time scale (500 $\mathrm{MHz}, 298 \mathrm{~K})$. This slow flipping rate enables the direct observation of the two $\mathrm{CB}$ portals existing in distinctly different chemical environments. ${ }^{22,28,39,41}$ Signal splitting is observed throughout the titration of the guest into a $\mathrm{CB}[7]$ solution, leading to quantitative splitting at a ratio of $1: 2$, which confirms the stoichiometric formula of this $\mathrm{CB}[7]$ complex as $\mathrm{G}_{1}-\mathrm{CB}[7]_{2}$. Thus, each Ant910Me molecule is readily isolated in a monomeric state in aqueous solution when complexed by two $\mathrm{CB}[7]$ macrocycles.

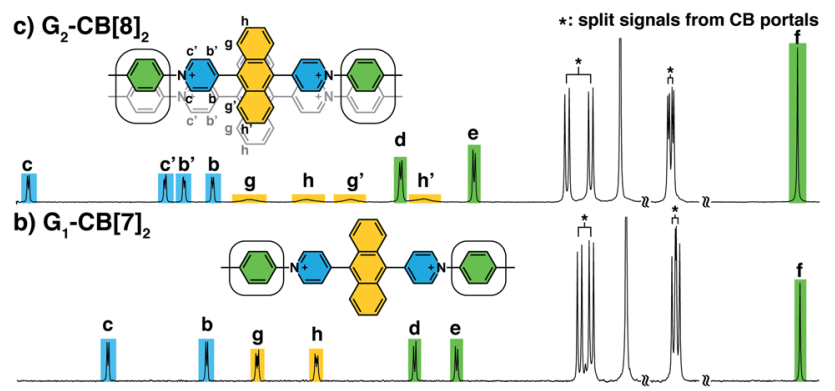

a) Ant910Me (G)

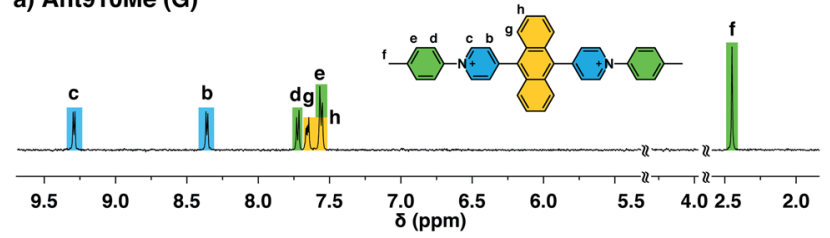

Fig. $2{ }^{1} \mathrm{H}$ NMR spectra $(500 \mathrm{MHz})$ of (a) Ant910Me (G), and (b) its complexation with 2 equiv. of $\mathrm{CB}[7]\left(\mathrm{G}_{1}-\mathrm{CB}[7]_{2}\right)$, or (c) with 1 equiv. of $\mathrm{CB}[8]\left(\mathrm{G}_{2}-\mathrm{CB}[8]_{2}\right)$ in $\mathrm{D}_{2} \mathrm{O}$ with a uniform guest concentration of 100 $\mu \mathrm{M}$ at $298 \mathrm{~K}$. The $\mathrm{Cl}^{-}$counterions are omitted for clarity.

\section{$2.2 \mathrm{G}_{2}-\mathrm{CB}[8]_{2}$ : preorganized $\pi$-stacked dimers}

Upon titration of Ant910Me into a solution of $\mathrm{CB}[8]$ (Fig. 2c), splitting of the $\mathrm{CB}[8]$ protons are observed as well as the upfield shift of $\mathrm{H}^{\mathrm{d}, \mathrm{e}, \mathrm{f}}$. Both observations suggest that the $\mathrm{CB}[8]$ molecules remain at the tolyl moieties, with a slow flipping rate and asymmetric portal environment. Careful analysis of the signal splitting and proton integration confirms a binding stoichiometry of " $1: 1$ ", therefore, this $\mathrm{CB}[8]$-mediated complex contains an equal number of hosts and guests. As elaborated in a previous work, ${ }^{28}$ this complex cannot be a $1: 1$ binary complex as $\mathrm{CB}[8]$-mediated binary complexes exhibit much faster dynamics. An elongated polymeric $\mathrm{G}_{n}-\mathrm{CB}[8]_{n}$ complex $(n=$ $1,2,3 \ldots)$, fabricated from the sequential stacking of tolyl groups ${ }^{23}$ is also not possible as the head-to-tail alignment of two tolyl groups would result in a symmetric portal environment contrary to the observed splitting. Therefore, the most probable binding mode is a $2: 2$ complex $\left(\mathrm{G}_{2}-\mathrm{CB}[8]_{2}\right)$, as illustrated in Fig. 2c, where two fluorescent molecules are constrained to overlap with each other. In this binding mode, the tolyl groups are head-to-head, thus resulting in an asymmetric portal environment for each $\mathrm{CB}[8]$. The observed slow flipping rate of $\mathrm{CB}[8]$ and the signal splitting is explained by the tightly filled $\mathrm{CB}[8]$ cavities as well as the electrostatic interactions between multiple positive charges on one side of the CB portals.

We have learned from previous works ${ }^{28,30,39,41,42}$ that the diffusion coefficient $(D)$ of a $\mathrm{CB}[n]$-mediated complex is primarily determined by the number of $\mathrm{CB}$ macrocycles existing in the complex. Therefore, the formation of $\mathrm{G}_{2}-\mathrm{CB}[8]_{2}$ is further confirmed through a semi-quantitative analysis of $D$ via DOSY experiments. As shown in Fig. 3a and Table $\mathrm{S} 1, \uparrow D$ values of unbound guests (G) in aqueous solution range from 3.49 to 4.38 , showing a standard deviation (SD) of 0.3. A much narrower distribution is observed for $\mathrm{CB}[8]$-mediated complexes, ranging from 1.95 to 2.07 with a SD of 0.04 (Fig. 3a). These $D$ values are much smaller than that of free $\mathrm{CB}[8](D=3.11)$ and typical
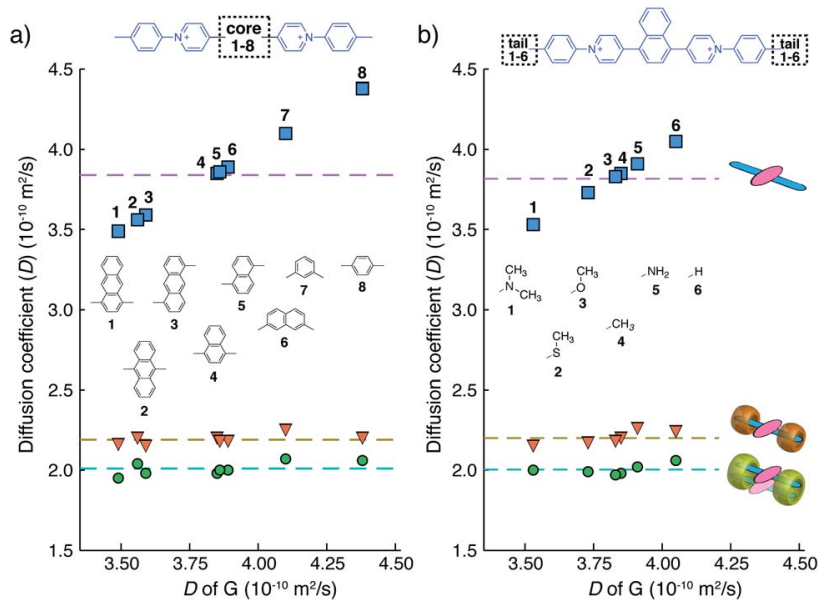

Fig. 3 Diffusion coefficients obtained from DOSY NMR for $G$ (rectangles), $\mathrm{G}_{1}-\mathrm{CB}[7]_{2}$ (triangles), and $\mathrm{G}_{2}-\mathrm{CB}[8]_{2}$ (circles) with variation in (a) fluorophore cores or (b) substituted tails. $\left(\mathrm{D}_{2} \mathrm{O}\right.$ solution with a uniform guest concentration of $100 \mu \mathrm{M}$ at $298 \mathrm{~K}$.) The $\mathrm{Cl}^{-}$counterions are omitted for clarity. 
binary complexes such as dzpy ${ }_{1}-\mathrm{CB}[8]_{1}(D=3.04) .{ }^{41}$ However, the $D$ values measured here are almost the same as for the $2: 2$ complexes produced by diarylviologen derivatives ${ }^{28,41}$ such as $\left(\mathrm{VNMe}_{2}\right)_{2}-\mathrm{CB}[8]_{2} \quad(D=2.01)$. Therefore, the DOSY data fully supports the formation of a $\mathrm{G}_{2}-\mathrm{CB}[8]_{2}$ complex involving the stacking of two fluorescent molecules held together by two $\mathrm{CB}[8]$ hosts.

The relative orientation of the two fluorophores with respect to each other is probed by proton and NOESY NMR in this dimeric system. As the proton spectrum recorded for $\mathrm{G}_{2}-\mathrm{CB}[8]_{2}$ ( $\mathrm{G}=$ Ant910Me) exhibits more complicated signal splittings than that of $\mathrm{G}_{1}-\mathrm{CB}[7]_{2}$, COSY NMR (Fig. S2 $\dagger$ ) is used to identify each proton. Both the pyridinium and anthracenyl protons in this $2: 2$ complex split into two sets of equivalent peaks corresponding to $\mathrm{H}^{\mathrm{b}, \mathrm{b}^{\prime}, \mathrm{c}, \mathrm{c}^{\prime}}$ and $\mathrm{H}^{\mathrm{g}, \mathrm{g}^{\prime}, \mathrm{h}, \mathrm{h}^{\prime}}$ in Fig. 2c. The observation of two sets of signals suggests (i) a slow dynamic process and (ii) a certain asymmetry existing for the most probable configuration of the $\mathrm{G}_{2}-\mathrm{CB}[8]_{2}$ complex, which is consistent with a cofacial stacking and partial overlap of the two aromatic fluorophores as illustrated in Fig. 2c. Partial overlap of the two fluorophores with a slippage along their extended axis will result in one set of equivalent protons lying on top of or below an aromatic ring of the other molecule, while the other set of equivalent protons does not. The first set of equivalent protons are expected to display signals in a higher-field region on account of shielding by aromatic ring currents, compared to the latter set of equivalent protons, which is consistent with the observation of significantly lowered chemical shifts for $\mathrm{H}^{\mathrm{g}^{\prime}, \mathrm{h}^{\prime}}$ compared to $\mathrm{H}^{\mathrm{g}, \mathrm{h}}$. Similarly, the difference observed between $\mathrm{H}^{\mathrm{c}, \mathrm{b}}$ and $\mathrm{H}^{\mathrm{c}^{\prime}, \mathrm{b}^{\prime}}$ is interpreted as two sets of protons that reside in different shielding and deshielding environments arising from the $\mathrm{CB}[8]$ portal.

The partial overlap of two aromatic fluorophores is also supported by the cross-correlation signals observed in NOESY NMR, which reveals the relative position of protons located in space. Proton $\mathrm{H}^{\mathrm{b}^{\prime}, \mathrm{c}^{\prime}}$ (Fig. $\mathrm{S} 3 \dagger$ ), for instance, exhibits an intense cross-correlation with all anthracenyl signals $\left(\mathrm{H}^{\mathrm{g}, \mathrm{g}^{\prime}, \mathrm{h}, \mathrm{h}^{\prime}}\right)$, whereas $\mathrm{H}^{\mathrm{b}, \mathrm{c}}$ can only "feel" protons that are closer to the pyridinium protons, i.e. $\mathrm{H}^{g, g^{\prime}}$. This observation is consistent with the partial-overlap and stacking of the fluorophores where $\mathrm{H}^{\mathrm{b}^{\prime}, \mathrm{c}^{\prime}}$ rather than $\mathrm{H}^{\mathrm{b}, \mathrm{c}}$ are closer to $\mathrm{H}^{\mathrm{h}, \mathrm{h}^{\prime}}$ in space (Fig. $2 \mathrm{c}$ and $\mathrm{S} 3 \mathrm{H}^{\dagger}$ ).

\subsection{Photophysics of the dimeric and monomeric Ant910Me}

The well-resolved NMR spectrum of $\mathrm{G}_{2}-\mathrm{CB}[8]_{2}$ suggests that these complexes exist as discrete preorganized fluorescent dimers in aqueous solution without forming any larger aggregates. This is because the two $\mathrm{CB}[8]$ macrocycles mechanically block the interaction between multiple dimers. It also leads to a substantial change in the photophysical properties of Ant910Me upon complexation with $\mathrm{CB}[8]$ to $\mathrm{G}_{2}-\mathrm{CB}[8]_{2}$.

As shown in Fig. $4 \mathrm{a}-\mathrm{c}$ and Table 1, the anthracenyl moiety in $\mathrm{G}_{2}-\mathrm{CB}[8]_{2}$ exhibits a bathochromic shift of its absorption maximum $\left(\lambda_{\text {abs }}=469 \mathrm{~nm}\right)$ by over $50 \mathrm{~nm}$ compared to monomeric Ant910Me $\left(\lambda_{\text {abs }}=409 \mathrm{~nm}\right)$ in $\mathrm{G}_{1}-\mathrm{CB}[7]_{2}$ and unbound Ant910Me in pristine solution $\left(\lambda_{\text {abs }}=419 \mathrm{~nm}\right)$ (all compared at a concentration of $15 \mu \mathrm{M})$. The emission maximum of $\mathrm{G}_{2}-\mathrm{CB}[8]_{2}$
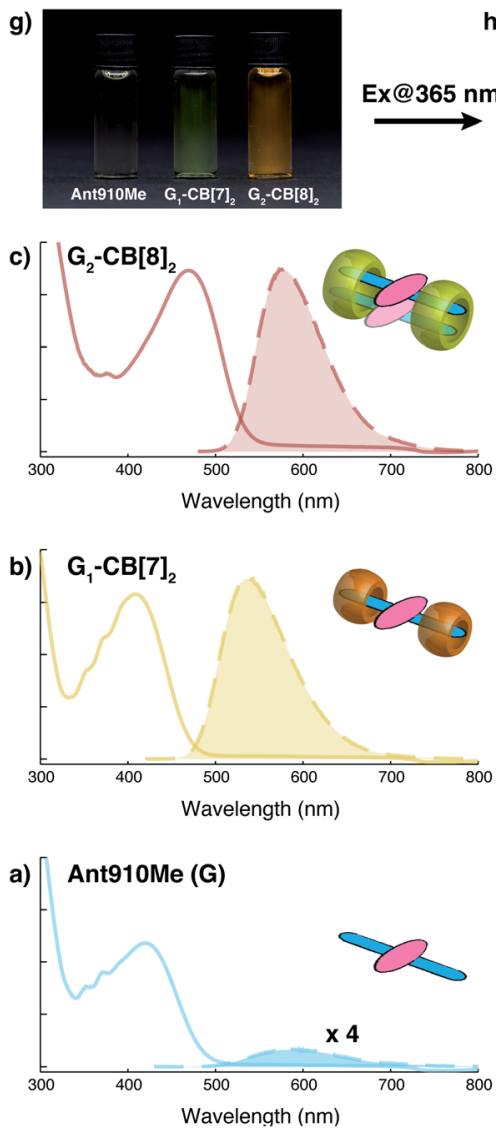
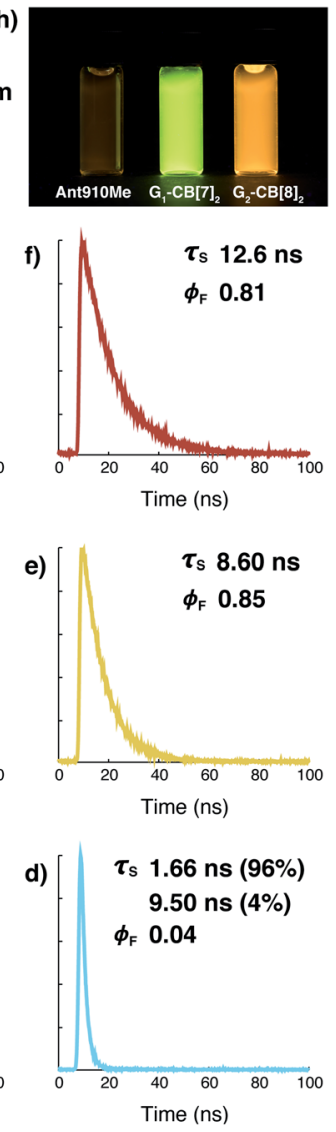

Fig. 4 Steady-state absorption (solid line) and emission (dash line with filling color) spectra of (a) Ant910Me (G), and (b) its $G_{1}-C B[7]_{2}$ complex, and (c) $\mathrm{G}_{2}-\mathrm{CB}[8]_{2}$ complex, whose time-dependent fluorescence decay is displayed in (d), (e), (f), respectively, along with corresponding lifetime $\left(\tau_{\mathrm{s}}\right)$ and fluorescence quantum yield $\left(\phi_{\mathrm{F}}\right)$ results. Aqueous solution of each species with a uniform guest concentration of $15 \mu \mathrm{M}$ is tested at $298 \mathrm{~K}$. The intensity is not normalized but scaled up by the same factor except the emission of $G$ which is enlarged by an additional 4 times for a clear vision. Quantified data can be found in Table 1. Photographs of each species with a guest concentration of 20 $\mu \mathrm{M}$ before $(\mathrm{g})$ and after (h) photoexcitation at $365 \mathrm{~nm}$.

$\left(\lambda_{\mathrm{em}}=578 \mathrm{~nm}\right)$ is also red-shifted relative to that of $\mathrm{G}_{1}-\mathrm{CB}[7]_{2}$ $\left(\lambda_{\mathrm{em}}=537 \mathrm{~nm}\right)$, although Ant910Me in pristine solution exhibits the most bathochromic shift in emission $\left(\lambda_{\mathrm{em}}=595\right.$ $\mathrm{nm})$.

After photoexcitation, fluorescence decay as well as the corresponding lifetime are recorded from time-correlated single photon counting (TCSPC) experiments, Fig. 4d-f. Excited anthracenyl dimers in the $\mathrm{G}_{2}-\mathrm{CB}[8]_{2}$ complexes display an excimer-like state that exhibits a lifetime $\left(\tau_{\mathrm{s}}\right)$ of $12.6 \mathrm{~ns}$, which is much longer than $\tau_{\mathrm{s}}$ of $8.6 \mathrm{~ns}$ for its monomeric counterpart in $\mathrm{G}_{1}-\mathrm{CB}[7]_{2}$. A biexponential decay is observed for Ant910Me in pristine solution measured at the same concentration $(15 \mu \mathrm{M})$ as that in $\mathrm{G}_{1}-\mathrm{CB}[7]_{2}$ and $\mathrm{G}_{2}-\mathrm{CB}[8]_{2}$, showing $96 \%$ of the intensity is due to a short-lived component of $1.66 \mathrm{~ns}$ and $4 \%$ arising from a long-lived component of $9.50 \mathrm{~ns}$.

Fluorescence quantum yields $\left(\phi_{\mathrm{F}}\right)$ for each species are measured by an absolute method using an integrating sphere. 
Table 1 Photophysical properties of studied fluorescent molecules and their CB[7]- or CB[8]-mediated complexes in aqueous solution at $298 \mathrm{~K}^{a}$

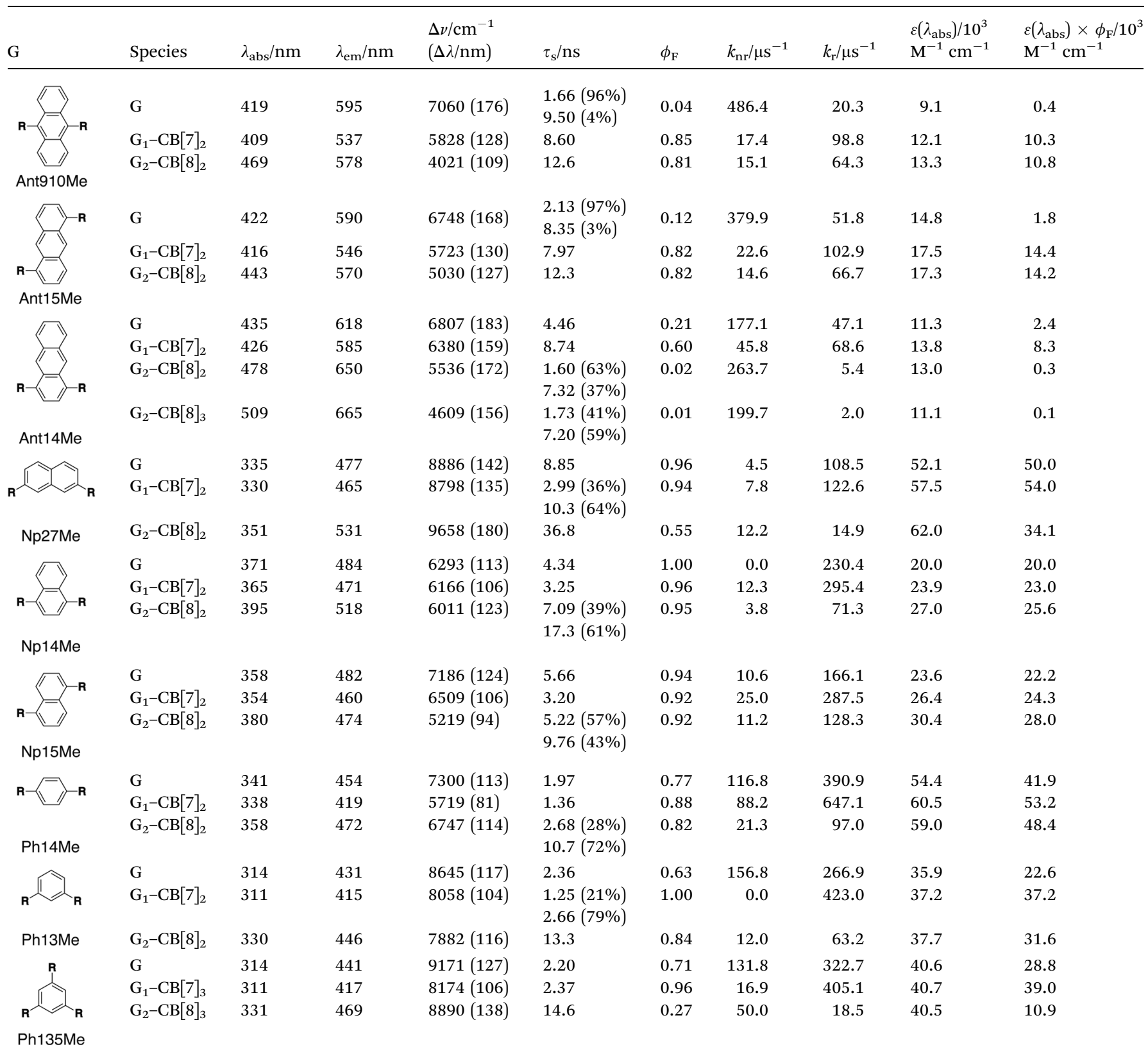

${ }^{a} \Delta \nu$ : wavenumber difference between $\lambda_{\mathrm{abs}}$ and $\lambda_{\mathrm{em}}$ ("Stokes shift"); $\varepsilon\left(\lambda_{\mathrm{abs}}\right)$ : molar absorption coefficient; $\phi_{\mathrm{F}}$ : fluorescence quantum yield; $\varepsilon\left(\lambda_{\mathrm{abs}}\right) \times \phi_{\mathrm{F}}$ : emission brightness.

The emission of Ant910Me is significantly quenched in a pristine solution with a $\phi_{\mathrm{F}}$ of 0.04 , whereas its fluorescence intensity is dramatically enhanced upon complexation with either $\mathrm{CB}[7]$ or $\mathrm{CB}[8]$, showing a $\phi_{\mathrm{F}}$ of 0.85 for $\mathrm{G}_{1}-\mathrm{CB}[7]_{2}$ and a $\phi_{\mathrm{F}}$ of 0.81 for $\mathrm{G}_{2}-\mathrm{CB}[8]_{2}$ (Table 1).

The negligible quantum yield, bimodal decay, and red-shifted emission in a pristine solution of Ant910Me all suggest a certain extent of aggregation in aqueous solution, whose photophysical properties are highly concentration-dependent. On the other hand, complexation with $\mathrm{CB}[7]$ or $\mathrm{CB}[8]$ ensures a dispersion of discrete fluorophores in solution in either monomeric or dimeric fashion, respectively. It is known that the polarity of $\mathrm{CB}$ cavities is lower than that of water, which will also affect photophysical properties of dye molecules. ${ }^{19,43}$ Therefore, in the following discussion, a comparison is made between $\mathrm{CB}[7]-$ and $\mathrm{CB}[8]$-mediated complexes on account of their similar cavity polarities. A comparison between $\mathrm{G}_{1}-\mathrm{CB}[7]_{2}$ and $\mathrm{G}_{2}-\mathrm{CB}[8]_{2}$ of Ant910Me shows that the stacking of anthracenyl moieties as a dimer, relative to monomer, exhibits (1) a significant bathochromic shift in absorption and emission, (2) an elongated 
excited-state lifetime, and (3) comparably high fluorescence efficiency.

\subsection{Applicability and flexibility of the modular strategy}

Although individual cases have demonstrated photophysical changes upon complexation with $\mathrm{CB}[8],,^{31,35,39,44-47}$ the beauty and power of this work stems from the simple modular design. As illustrated in Fig. 1a, any selected fluorophore can be readily inserted between clamping modules, resulting in its monomeric or dimeric species through complexation with $\mathrm{CB}[7]$ or $\mathrm{CB}[8]$, respectively.

Following this design strategy, a further eight fluorescent molecules were successfully synthesized, with similar topology to Ant910Me but with systematic variation in their structures. For example, the fluorophore cores are augmented between phenyl, naphthyl, and anthracenyl. Alternatively, the alignment between the two clamping modules is altered. While several derivatives exhibit both clamping modules in-line with one another (Ph14Me, Np14Me, Ant910Me, and Ant14Me) others have clamping modules that are not in-line but remain parallel to each other such as Np15Me and Ant15Me, or are no longer aligned in a parallel manner but with an angle $<180^{\circ}$ (Ph13Me and Np27Me). Finally, one can readily add additional clamping motifs around the fluorophore core moiety, as demonstrated in the triply clamped systems Ph135Me and Ant14Me.

Results from ${ }^{1} \mathrm{H}$ NMR and DOSY, as shown in Fig. 3a and in the ESI (Table S1 and Fig. S1-S17†), demonstrates that these fluorescent molecules all perform in a manner similar to Ant910Me. Despite their structural variation, they all generate a monomeric fluorophore in the presence of $\mathrm{CB}$ [7] and dimeric stacking of fluorophores with $\mathrm{CB}[8]$. As $\mathrm{CB}[7]$ and $\mathrm{CB}[8]$ only bind the clamping modules (i.e. tolyl pyridinium moieties), choice of the fluorophores is no longer limited by the size and shape of the macrocycle cavities. Large fluorophores such as anthracenyl derivatives, which to date have only been shown to complex $\mathrm{CB}[7]$ or $\mathrm{CB}[8]$ along their principal symmetry axis, are easily incorporated using this strategy regardless of their substitution pattern. Moreover, small aromatic rings like phenyl moieties, whose binding is extremely dynamic inside a single $\mathrm{CB}[8]$ cavity, are now readily immobilized and constrained within a $2: 2$ complex.

\subsection{Photophysical properties}

In terms of photophysical properties, most fluorescent molecules also behave similarly to Ant910Me, with the exception of a few outliers that are discussed later in detail.

Preorganized ground-state dimers are readily produced by the formation of $\mathrm{G}_{2}-\mathrm{CB}[8]_{2}$ in aqueous solution, corresponding to a considerable bathochromic shift in the absorption band (Fig. 5 and Table 1). An excimer-like emission with a broadened and structureless profile is observed for all fluorophores in their $\mathrm{G}_{2}-\mathrm{CB}[8]_{2}$ systems, exhibiting a red-shift in their emission maximum relative to their monomeric form in $\mathrm{G}_{1}-\mathrm{CB}[7]_{2}$ systems. Solutions of $\mathrm{G}_{2}-\mathrm{CB}[8]_{2}$ compared to their $\mathrm{G}_{1}-\mathrm{CB}[7]_{2}$ counterparts exhibit a smaller rate constant for non-radiative deactivation $\left(k_{\mathrm{nr}}\right)$, which corresponds to their observed elongated excited-state lifetime as well as comparably high quantum yields. Molar absorption coefficients for all fluorophores are slightly increased upon complexation with either $\mathrm{CB}[7]$ or $\mathrm{CB}[8]$ (Table 1) along with their high quantum yield, leading to reasonably high brightness $\left(\varepsilon \times \phi_{\mathrm{F}}\right)$ in aqueous solution. ${ }^{\mathbf{4 8}}$ Considering their long fluorescence lifetimes, $\mathrm{G}_{2}-\mathrm{CB}[8]_{2}$ complexes in general should be promising candidates for timegated imaging for biological systems.
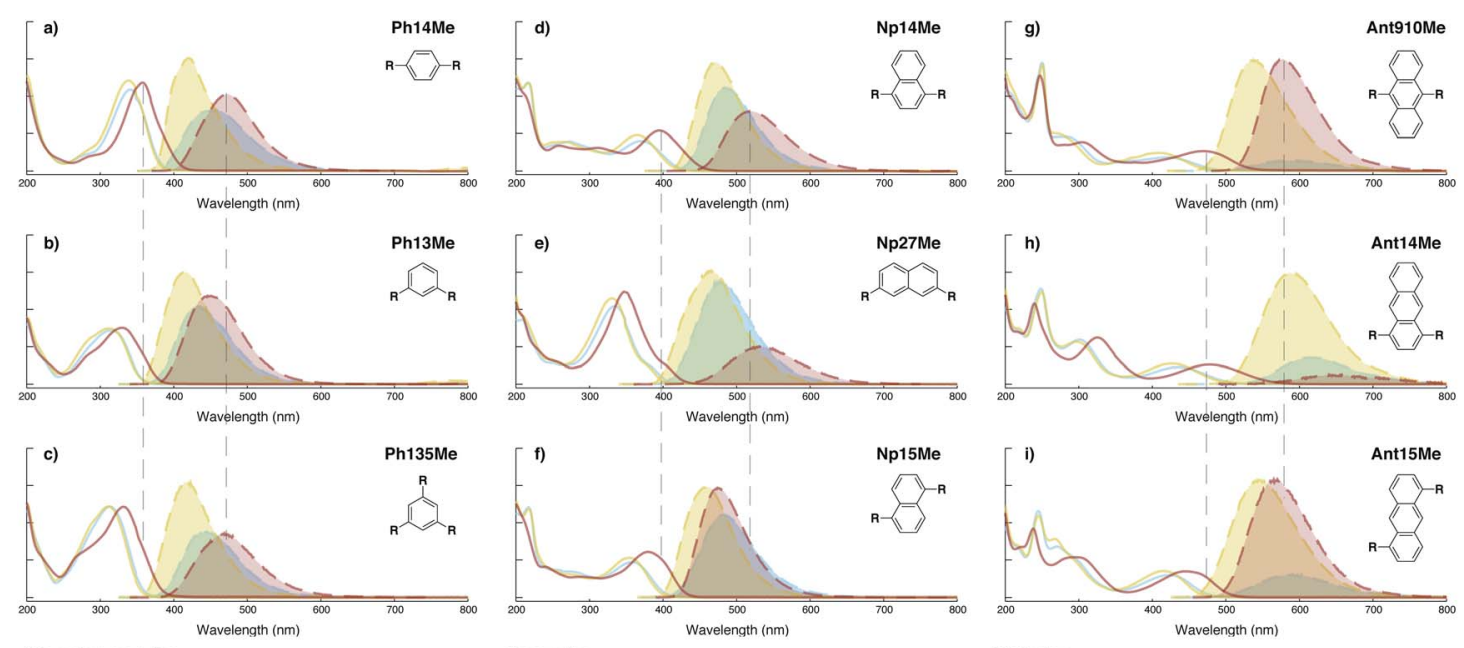

Clamping modules:

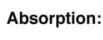

G $\quad \mathrm{G}_{1}-\mathrm{CB}[7]_{2}$ or $\mathrm{G}_{1}-\mathrm{CB}[7]_{3} \quad \mathrm{G}_{2}-\mathrm{CB}[\mathrm{B}]_{2}$ or $\mathrm{G}_{2}-\mathrm{CB}[8]_{3}$

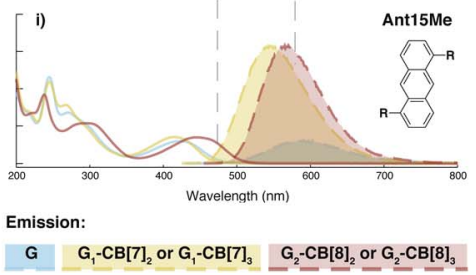

Fig. 5 Steady-state absorption (solid line) and emission (dash line with filling color) spectra of non-associated fluorescent molecules (G in blue), and their $\mathrm{CB}[7]$-mediated complexes $\left(\mathrm{G}_{1}-\mathrm{CB}[7]_{2}\right.$ or $\mathrm{G}_{1}-\mathrm{CB}[7]_{3}$ in yellow) as well as $\mathrm{CB}[8]$ mediated complexes $\left(\mathrm{G}_{2}-\mathrm{CB}[8]_{2}\right.$ or $\mathrm{G}_{2}-\mathrm{CB}[8]_{3}$ in red). The study covers the derivatives of three types of fluorophores including phenyl such as (a) Ph14Me, (b) Ph13Me, (c) Ph135Me, naphthyl such as (d) Np14Me, (e) Np27Me, (f) Np15Me, and anthracenyl such as (g) Ant910Me, (h) Ant14Me, (i) Ant15Me. Aqueous solutions of each species are tested under a uniform guest concentration at $298 \mathrm{~K}$. The intensity is not normalized but scaled up by the same factor in most cases. Quantified data can be found in Table 1 . 
Fluorescent molecules that contain anthracenyl cores all exhibit a low quantum yield in solution with a lifetime much shorter than that in their $\mathrm{CB}[7]$ - or $\mathrm{CB}[8]$-mediated complexes. The discrete monomeric species of Ant910Me, Ant15Me, and Ant14Me in their corresponding $\mathrm{G}_{1}-\mathrm{CB}[7]_{2}$ complexes all display a lifetime around $8 \mathrm{~ns}$ (Table 1 ), similar to the lifetime $\left(\tau_{\mathrm{s}}\right)$ of 9,10-diphenylanthracene (DPA), a typical anthracenyl standard. ${ }^{49}$ This recovery of lifetime to a value similar to DPA implies that the fluorescence of these $\mathrm{CB}$ [7] complexes is mainly contributed by their anthracenyl cores. In a solution of only free molecules (without the presence of CB), the excited anthracenyl cores are deactivated through certain pathways as evidence from the observed quenching of fluorescence. In particular, a typical deactivation pathway would be the photoinduced electron transfer (PET) from the anthracenyl core to $\pi$-deficient pyridinium moieties. ${ }^{\mathbf{5 0 , 5 1}}$ However, the significant recovery of emission after complexation suggests that these deactivation pathways are forbidden or are at least largely restricted in both the $G_{1}-C B[7]_{2}$ and $G_{2}-C B[8]_{2}$ complexes. Quantum yields of the naphthyl and phenyl species are generally large (0.9-1.0 for $\mathrm{Np}$, 0.6-1.0 for $\mathrm{Ph}$ ) contrary to anthracenyl analogues, regardless of complexation, implying that PET from these two fluorophore cores to pyridinium moieties is not efficient.

Systematic variation in the alignment between the clamping modules also affects their photophysical properties. Np15Me and Ant15Me, with two parallel clamping modules that are not aligned, exhibit a red-shift in emission, which is not as large as for other species (Fig. 5) upon forming $\mathrm{G}_{2}-\mathrm{CB}[8]_{2}$ complexes. This non-aligned connectivity may force the two fluorophores to stack in a less $J$ aggregate-like fashion. ${ }^{52}$ When the clamping modules are non-parallel, the $\mathrm{G}_{2}-\mathrm{CB}[8]_{2}$ of $\mathbf{N p 2 7 M e}$ displays a quantum yield of 0.55 , which is almost half the value of the other naphthyl homologues (Table 1). However, this species exhibits a distinctively long-lived excited state with a $\tau_{\mathrm{s}}$ up to 37 ns. Similar results are observed in $\mathrm{CB}[8]$-mediated complexes of Ph13Me, which also possesses non-parallel clamping modules. The reduced fluorescence efficiency along with the elongated lifetime suggests that dimeric stacking in species with nonparallel clamping units may significantly suppress radiative pathways (i.e. see reduced $k_{\mathrm{r}}$ values in Table 1 ).

\subsection{Triple clamping}

Ph135Me is a more complex version of non-parallel clamping, which forms dimeric stacks through triple clamping, denoted $\mathrm{G}_{2}-\mathrm{CB}[8]_{3}$ (Fig. S16 $\dagger$ ). Triple non-parallel clamping leads to a further decrease in $k_{\mathrm{r}}$ (Table 1 ) compared to $\mathrm{G}_{2}-\mathrm{CB}[8]_{2}$ of Ph13Me, which results in a reduced quantum yield (0.27) of $\mathrm{G}_{2}-\mathrm{CB}[8]_{3}$ that is about one third of that of its $\mathrm{CB}[7]$-mediated complex, $\mathrm{G}_{1}-\mathrm{CB}[7]_{3}$. Besides suppressing the radiative pathway, triple clamping exhibits a concerted feature of multivalency ${ }^{53}$ further stabilizing the dimeric stacking of two phenyl moieties. In a mixture consisting of 4 equiv. of Ph135Me and 3 equiv. of $\mathrm{CB}[8]$, excess guest molecule does not result in statistical complexes such as $\mathrm{G}_{2}-\mathrm{CB}[8]_{2}$ and $\mathrm{G}_{2}-\mathrm{CB}[8]_{1}$ (Fig. S17 $\dagger$ ). Instead, Ph135Me molecules exist either as $\mathrm{G}_{2}-\mathrm{CB}[8]_{3}$ complexes or as a free guest in aqueous solution.
In addition to Ph135Me, which has three uniform clamping modules, Ant14Me with a protruding fluorophore core is also able to form a $\mathrm{G}_{2}-\mathrm{CB}[8]_{3}$ complex. Isothermal titration calorimetry and UV-Vis titration both confirm a binding stoichiometry of $2: 3$ (Fig. S12 $\dagger$ ). Its diffusion coefficient from DOSY NMR gives a $D$ value similar to that of $\mathrm{Ph} 135 \mathrm{Me}_{2}-\mathrm{CB}[8]_{3}$ (Table S1, Fig. S11 and S16 $\dagger$ ). Considering its T-shape topology, a third $\mathrm{CB}[8]$ in the Ant14Me $\mathrm{G}_{2}-\mathrm{CB}[8]_{3}$ complex binds with the two protruding, stacked anthracenyl cores. However, in contrast to Ph135Me, CB[8] complexation of Ant14Me (in excess) does not exhibit a self-sorting behavior. Addition of extra Ant14Me guest molecules gradually transforms the solution of $\mathrm{G}_{2}-\mathrm{CB}[8]_{3}$ into 2 : 2 complexes, in which two $\mathrm{CB}[8]$ macrocycles are bound with the two tolyl pyridinium moieties rather than the protruding anthracenyl cores (Fig. S10-S12 $\dagger$ ). This suggests that the affinity of $\mathrm{CB}[8]$ around the protruded binding site is substantially weaker than its binding with the clamping modules, which is confirmed by the ITC result in Fig. S12. $\dagger$

\subsection{Restricted intracomplex motion}

Dimeric fluorophore stacking in $\mathrm{G}_{2}-\mathrm{CB}[8]_{2}$ complexes generally exhibit an enhanced fluorescence efficiency, particularly in the case of employing anthracenyl motifs as cores. This observation implies that motion within the complex (intracomplex motion) of $\mathrm{G}_{2}-\mathrm{CB}[8]_{2}$ is extremely retarded and restricted, thus effectively suppressing deactivation pathways.

2.7.1 Interconversion dynamics quantified by VT-NMR. The NOESY spectrum of $\mathrm{G}_{2}-\mathrm{CB}[8]_{2}$ (Fig. S3†) shows that crosscorrelations between $\mathrm{H}^{\mathrm{g}}$ and $\mathrm{H}^{\mathrm{g}^{\prime}}$ as well as those between $\mathrm{H}^{\mathrm{h}}$ and $\mathrm{H}^{\mathrm{h}^{\prime}}$ are much more intense than correlations caused by ${ }^{3} J_{\mathrm{H}-\mathrm{H}}$ coupling for $\mathrm{H}^{\mathrm{g}}-\mathrm{H}^{\mathrm{h}}$ and $\mathrm{H}^{\mathrm{g}^{\prime}}-\mathrm{H}^{\mathrm{h}^{\prime}}$. As chemical exchange also contributes to NOESY signals, this observation implies the presence of a dynamic interconversion between two discrete states within the $\mathrm{CB}[8]$ complex. This is also the reason why anthracenyl and pyridinium proton signals in $\mathrm{G}_{2}-\mathrm{CB}[8]_{2}$ split into two sets of equally intense peaks (Fig. 2c) that are not observed in $\mathrm{G}_{1}-\mathrm{CB}[7]_{2}$ (Fig. 2b).

Interconversion between the two states is further confirmed and quantified by variable-temperature nuclear magnetic resonance spectroscopy (VT-NMR). As shown in Fig. 6a, four signals of $\mathrm{H}^{\mathrm{g}, \mathrm{g}^{\prime}, \mathrm{h}, \mathrm{h}^{\prime}}$ that correspond to two stacked anthracenyl cores broaden equally until coalescence is observed as the temperature rises from $278.6 \mathrm{~K}$ to $307.5 \mathrm{~K}$ on a high-field NMR spectrometer $(500 \mathrm{MHz})$. A subsequent increase of temperature from $306.2 \mathrm{~K}$ to 362.6 K on a low-field NMR spectrometer (200 MHz) (Fig. 6b) leads to a gradual merging of the four signals into two broad peaks, which later become sharper as the temperature increases. The transition of the $\mathrm{H}^{\mathrm{g}, \mathrm{g}^{\prime}, \mathrm{h}, \mathrm{h}^{\prime}}$ signals from the slow exchange limit to the fast exchange limit confirms the existence of a dynamic interconversion between two discrete states for the anthracenyl pair. By analysing the temperature-dependent linebroadening in the slow exchange limit ${ }^{54-56}$ (Fig. S19†), an activation energy of $43 \mathrm{~kJ} \mathrm{~mol}^{-1}$ is obtained for this interconversion.

As lowering the magnetic field is equivalent to severely heating the sample, the switch of VT-NMR from high-field to low-field enables us to witness and quantify a very slow 

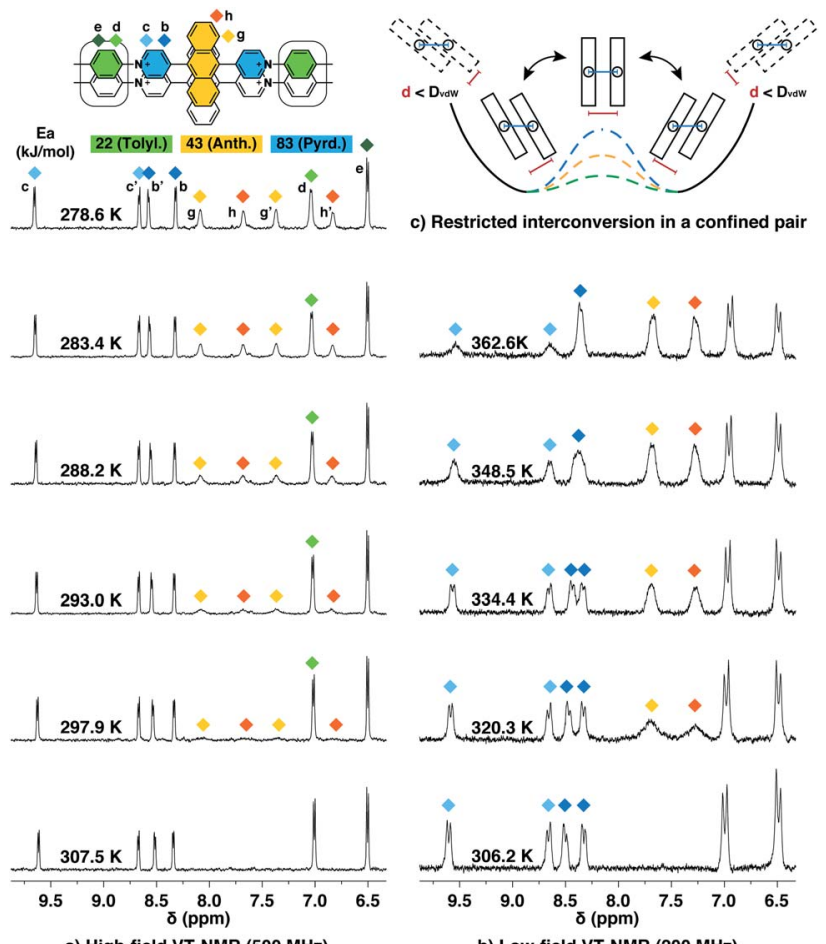

a) High-field VT-NMR (500 MHz)

b) Low-field VT-NMR (200 MHz)

Fig. 6 Variable-temperature ${ }^{1} \mathrm{H}$ NMR spectra of the Ant910Me ${ }_{2}-\mathrm{CB}[8]_{2}$ complex in $\mathrm{D}_{2} \mathrm{O}$ solution (a) with a temperature increased from $278.6 \mathrm{~K}$ to $307.5 \mathrm{~K}$ (top to bottom) recorded by high-field spectrometer $(500 \mathrm{MHz})$, and (b) with a temperature increased from $306.2 \mathrm{~K}$ to $362.6 \mathrm{~K}$ (bottom to top) recorded by low-field spectrometer ( $200 \mathrm{MHz}$ ), showing (c) restricted intracomplex rotations of tolyl, anthracenyl, and pyridinium

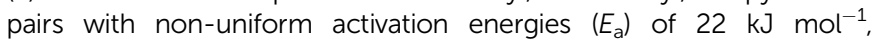
$43 \mathrm{~kJ} \mathrm{~mol}^{-1}$, and $83 \mathrm{~kJ} \mathrm{~mol}^{-1}$, respectively, which are analysed from the temperature-dependent line-broadening (Fig. S19 $\dagger$ ). The temperature of $500 \mathrm{MHz}$ and $200 \mathrm{MHz}$ spectrometers is calibrated by MeOD (D, 99.8\%) and ethylene glycol $\left(80 \%\right.$ in DMSO- $\left.d_{6}\right)$, respectively. The $\mathrm{Cl}^{-}$counterions are omitted for clarity.

exchange process such as that for the pyridinium pair in this study. In the spectra recorded by the high-field spectrometer (Fig. 6a), no significant line broadening is observed for pyridinium signals $\left(\mathrm{H}^{\mathrm{b}, \mathbf{b}^{\prime}, \mathbf{c}, \mathrm{c}^{\prime}}\right)$, whereas in the low-field VT-NMR, the signal broadening corresponding to an exchange in the slow limit is readily observed upon increase in temperature (Fig. 6b). The temperature-dependent signal broadening suggests an activation energy as large as $83 \mathrm{~kJ} \mathrm{~mol}^{-1}$ (Fig. S19†), implying a relatively slow interconversion of the pyridinium pair within the complex. The interconversion of protons in the stacked tolyl pair is already displayed in the fast exchange limit as demonstrated by the line sharpening of the $\mathrm{H}^{\mathrm{d}}$ signal as the temperature is increased in the high-field spectrometer, exhibiting a relatively small exchange barrier of $22 \mathrm{~kJ} \mathrm{~mol}^{-1}$ (Fig. 6a and S19†).

2.7.2 Intracomplex motion restricted in constrained dimers. Despite the covalent bonds between the anthracenyl, pyridinium, and tolyl moieties, different interconversion barriers are observed from VT-NMR indicating three separate dynamic processes. Therefore, these three distinct processes cannot be attributed to either the back and forth shuffling of the two fluorophores along the long axis of the complex or to the complexation/decomplexation process with $\mathrm{CB}[8]$ because these two processes require a simultaneous movement of all components at the same rate yielding uniform activation energies. Moreover, complexation/decomplexation must be slower than all three dynamic processes observed. This indicates that the $\mathrm{G}_{2}-\mathrm{CB}[8]_{2}$ complex is fairly "static" in aqueous solution and must remain complexed longer than the dynamics for pyridinium interconversion, which is around $30 \mathrm{~ms}$ at room temperature.

Molecular dynamic (MD) simulations of the Ant $910 \mathrm{Me}_{2}-\mathrm{CB}[8]_{2}$ complex in a cubic water box with $4000 \mathrm{H}_{2} \mathrm{O}$ molecules was carried out in order to evaluate the stability of this complex under ambient conditions (298 K, $1 \mathrm{~atm}$ ). The simulations indicate that the $\mathrm{Ant} 10 \mathrm{Me}_{2}-\mathrm{CB}[8]_{2}$ complex (ESI $\dagger$ video media) remains as a single entity during the whole simulation period $(>200 \mathrm{~ns})$ without decomplexation or significantly altering its structure. This result is consistent with the analysis by NMR, which shows that the dimeric stack of fluorophores is constrained and stabilized by the $\mathrm{CB}[8]$-mediated dual clamping. Moreover, the two stacked aromatic moieties, such as anthracenyl units, partially overlap one another and simultaneously rotate in a slow but coherent fashion during the MD simulation. For example, the two anthracenyl units (yellow) of the Ant $910 \mathrm{Me}_{2}-\mathrm{CB}[8]_{2}$ complex in Fig. 6 must rotate or swing around the central axis of the complex in a coupled manner, which we refer to here as intracomplex motion. Thus, the activation energy obtained represents the energy barrier for each intracomplex rotation.

The height of the energy barrier reflects the steric hindrance present around the "rotor". As exemplified by Ant910Me in Fig. 7, the rotation of the anthracenyl group is hindered by the presence of several pairs of adjacent protons between the anthracenyl core and pyridinium units. The rotation of the

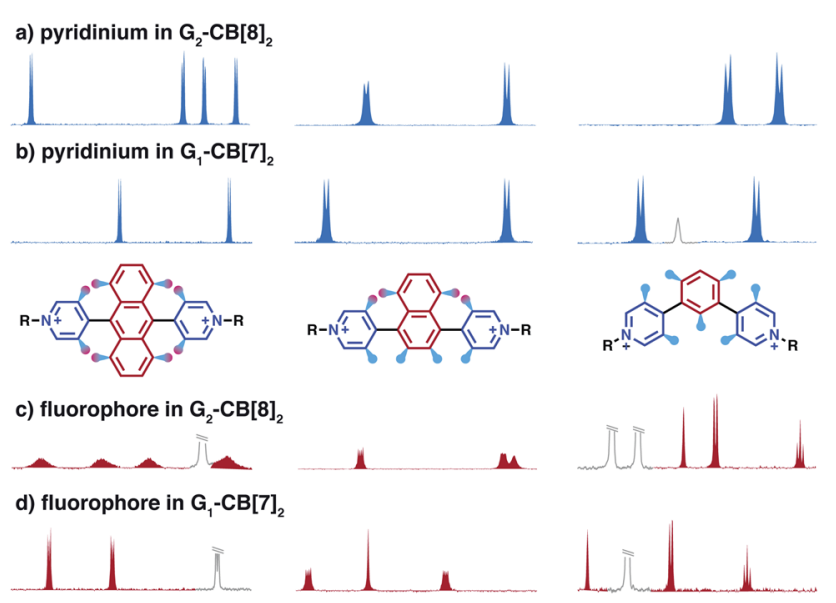

Fig. 7 Linewidth comparison between $G_{2}-C B[8]_{2}$ and $G_{1}-C B[7]_{2}$ complexes for fluorescent molecules (Ant910Me, Np14Me, Ph13Me) with a variation in the extent of proton exclusion. The difference in steric hindrance of each fluorescent molecule is readily reflected by the signal broadening or splitting observed from both fluorophore and pyridinium moieties in $\mathrm{G}_{2}-\mathrm{CB}[8]_{2}$, but cannot be distinguished by those in $\mathrm{G}_{1}-\mathrm{CB}[7]_{2}$, indicating that the steric hindrance is largely amplified in a dimeric stacked complex. 
pyridinium moieties, in addition to steric hindrance from the anthracenyl core, are also impeded by the $\mathrm{CB}[8]$ portals, thus showing the highest rotational barrier. On the other hand, rotation of the tolyl groups is not significantly influenced by CB portals as they mainly reside within the $\mathrm{CB}[8]$ cavities. Their motion is also not significantly retarded by neighboring pyridinium protons, which present less steric clash than those between the anthracenyl and pyridinium units. Therefore, the tolyl groups exhibit the lowest rotational barrier and their proton signals always fall within the fast exchange limit (Fig. 6). It is worth noting that "rotation" does not necessarily refer to a full rotation. In the case of Ant910Me, it is more likely that the two anthracenyl groups swing coherently within a limited angle on account of van der Waals repulsion (Fig. 6c), where the activation energy represents the steric hindrance for swinging between two degenerate states.

2.7.3 Steric hindrance amplified in $\mathrm{G}_{2}-\mathrm{CB}[8]_{2}$ complexes. It is worth highlighting that steric hindrance between adjacent aromatic moieties is significantly amplified in the stacked dimers $\left(\mathrm{G}_{2}-\mathrm{CB}[8]_{2}\right)$, which exhibit substantially slower dynamics than their monomeric counterparts $\left(\mathrm{G}_{1}-\mathrm{CB}[7]_{2}\right)$. As shown in Fig. 7, the fluorophore and pyridinium moieties within the three molecules Ant910Me, Np14Me, and Ph13Me should experience a different degree of steric hindrance consistent with the number of clashing, neighboring protons. However, this difference is not observed in their monomeric forms $\left(\mathrm{G}_{1}-\mathrm{CB}[7]_{2}\right)$ where proton signals attributed to pyridinium (Fig. 7b) and fluorophore units (Fig. 7d) are all sharp and are not split indicative of dynamics within the fast exchange limit.

In contrast, the dimeric complexes of these molecules $\left(\mathrm{G}_{2}-\right.$ $\mathrm{CB}[8]_{2}$ ) display significant differences in both their fluorophore and pyridinium components in their ${ }^{1} \mathrm{H}$ NMR spectra. As shown in Fig. 7a and c, proton signals of Ph13Me exhibit a narrow linewidth in the fast exchange limit for both the pyridinium and 1,3-phenyl groups, which is consistent with the fact that no severe steric clash exists in this molecule. However, signal broadening occurs with an increase of steric repulsion in the dimeric complex of Np14Me. Furthermore, proton signals from the anthracenyl and pyridinium groups in the dimeric complex of Ant910Me both fall into a slow exchange limit and split into two sets of peaks, which corresponds to much slower intracomplex motions. This observation stems from a further increase in steric hindrance and is amplified for the $\mathrm{G}_{2}-\mathrm{CB}[8]_{2}$ complexes as rotation of one moiety is not only retarded by covalently linked "neighbours" but also hindered by adjacent groups on the other stacked molecule. Careful comparison between the monomeric and dimeric systems verifies that formation of a constrained system largely restricts and slows down intracomplex motions in these dimers.

\subsection{Ground and excited states of $\pi$-stacked dimers}

2.8.1 Preorganized $\pi$-stacked ground-state dimer. The characteristic red-shift in emission and elongated excited-state lifetime (Fig. 4c and Table 1) suggest the formation of an excimer-like state for $\mathrm{G}_{2}-\mathrm{CB}[8]_{2}$ upon photoexcitation. However, the formation of the excimer-like state in $\mathrm{G}_{2}-\mathrm{CB}[8]_{2}$ complexes is quite different from those formed by pyrene derivatives or covalently linked pseudo-dimers., ${ }^{2,48,57}$ In such cases, the generation and decay of an excimer or excimer-like state involves the excitation of one single fluorophore followed by a diffusion-controlled interaction with a second ground-state fluorophore and ends up with relaxation towards the ground state. ${ }^{58}$ Therefore, the absorption band is often similar to that of a monomeric fluorophore as the excitation is firstly applied to a single molecule. ${ }^{2}$

In the case of $\mathrm{G}_{2}-\mathrm{CB}[8]_{2}$ (Fig. 5), however, a considerable bathochromic shift is generally observed in its steady-state absorption spectrum. Particularly, the vibronic progression is absent in the absorption of Ant910 $\mathrm{Me}_{2}-\mathrm{CB}[8]_{2}$ (Fig. 4c) indicating a strong coupling and effective delocalization of $\pi$-electrons between the dimeric anthracenyl moieties at their ground states. This preorganized $\pi$-stacked ground-state dimer is excited as a precoupled entity to an excimer-like state, which is different from an excited monomer and, more importantly, does not require an additional diffusion-controlled process after photoexcitation. On the other hand, the excited $\mathrm{G}_{2}-\mathrm{CB}[8]_{2}$ complex will not exhibit an energy dissipation as significantly as during the formation of conventional excimers. This explains why Ant910Me in the $\mathrm{G}_{2}-\mathrm{CB}[8]_{2}$ complex exhibits a Stokes shift (wavenumber difference between $\lambda_{\text {abs }}$ and $\lambda_{\text {em }}$ ) of $4012 \mathrm{~cm}^{-1}$ $(109 \mathrm{~nm})$ smaller than the value of $5828 \mathrm{~cm}^{-1}(128 \mathrm{~nm})$ in its $\mathrm{G}_{1}-\mathrm{CB}[7]_{2}$ complex (Table 1 ). Due to the absence of diffusioncontrolled steps in their excited state, one expects a monoexponential fluorescence decay at pico- and nano-second timescale for $\mathrm{G}_{2}-\mathrm{CB}[8]_{2}$ complexes after photoexcitation, contrary to the bimodal decay of conventional excimers. ${ }^{58}$

2.8.2 Mono-exponential decay of excited $G_{2}-\mathbf{C B}[8]_{2}$. A mono-exponential fluorescence decay is indeed observed for the Ant $910 \mathrm{Me}_{2}-\mathrm{CB}[8]_{2}$ complex in TCSPC measurements (Fig. 4 and Table 1) and is further validated by time-resolved spectroscopies.

Femtosecond (fsTA) and nanosecond (nsTA) transient absorption were employed to monitor the dynamic relaxation of both $\mathrm{G}_{2}-\mathrm{CB}[8]_{2}$ and $\mathrm{G}_{1}-\mathrm{CB}[7]_{2}$ complexes of Ant910Me after photoexcitation (Fig. S20-S25, Table S2†). As shown in Fig. 8a and $\mathrm{b}$, two species are clearly detected in the excited state from fsTA for Ant910Me $\mathrm{Me}_{2}-\mathrm{CB}[8]_{2}$. Both exhibit a spectral feature of ground state bleaching (GSB) from $431 \mathrm{~nm}$ to $498 \mathrm{~nm}$ overlapping with an excited state absorption (ESA) from $431 \mathrm{~nm}$ to $800 \mathrm{~nm}$ and a stimulated emission (SE) from $573 \mathrm{~nm}$ to $654 \mathrm{~nm}$. Upon photoexcitation, the first species A relaxes to species $\mathrm{B}$ with a fairly short lifetime of $3.6 \pm 0.3$ ps (Fig. 8c, d and S21 $\dagger$ ), and then back to its ground state with a lifetime of $12.9 \pm 0.4 \mathrm{~ns}$ (Fig. 8e and f), consistent with the value of 12.6 ns measured from TCSPC (Table 1). Species B exhibits a similar ESA profile as species A except a slight red-shift in its absorption maximum (Fig. 8b), which suggests that the evolution from A to B with a picosecond time constant probably corresponds to excited state solvation..$^{59}$ In addition to solvation, the excited complex relaxes back to its ground state in a mono-exponential manner without observing other competitive pathways. It is worth mentioning that the excited state absorption spectra of 

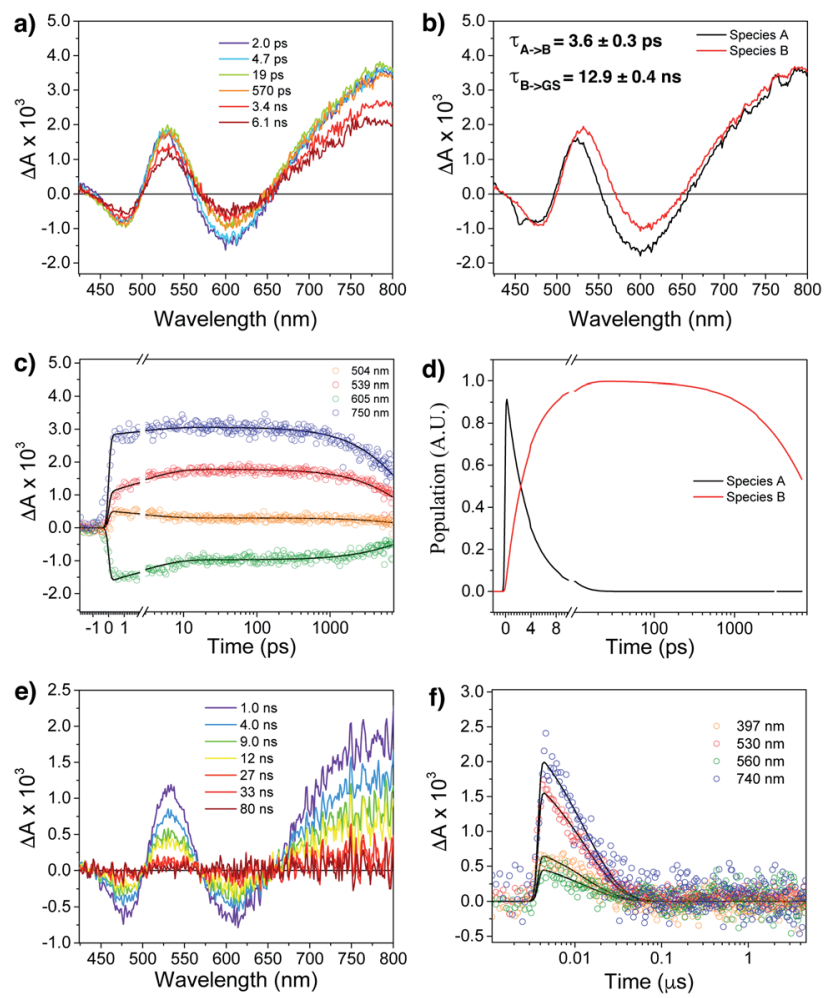

Fig. 8 Femtosecond transient absorption spectra (fsTA) of (a) Ant $910 \mathrm{Me}_{2}-\mathrm{CB}[8]_{2}$ complexes in $\mathrm{D}_{2} \mathrm{O}$ at $298 \mathrm{~K}$ following a laser pulse of $414 \mathrm{~nm}(1 \mu \mathrm{J}$ per pulse). Fitting from the raw data using a kinetic model A $\rightarrow$ B $\rightarrow$ GS gives (b) species associated spectra, (c) wavelength fitting, and (d) model population kinetics. (e) Nanosecond transient absorption spectra (nsTA) of species $B$ and ( $f$ ) its kinetic fitting at selected wavelength using mono-exponential model.

Ant910Me ${ }_{2}-\mathrm{CB}[8]_{2}$ (Fig. 8a) are quite broad suggesting a strong coupling also existing in the excited states.

Ant910 $\mathrm{Me}_{1}-\mathrm{CB}[7]_{2}$ after photoexcitation (Fig. S23†) exhibits an absorption maximum at around $475 \mathrm{~nm}$ in its ESA profile, which is much smaller than that of Ant910 $\mathrm{Me}_{2}-\mathrm{CB}[8]_{2}$ at around $530 \mathrm{~nm}$ (Fig. 8b). This observation confirms that $\mathrm{G}_{2}-\mathrm{CB}[8]_{2}$ complexes are directly pumped up to the excited state of precoupled dimers rather than the excited state of monomers. It is worth noticing that several species are detected (Fig. S23 and $\mathrm{S} 24 \dagger$ ) for Ant910Me ${ }_{1}-\mathrm{CB}[7]_{2}$ upon photoexcitation, which also includes a small amount of long-lived species with a time constant of $6 \pm 1 \mu \mathrm{s}$. This long-lived species should come from a triplet state, as its lifetime increases significantly ( $>340 \mu \mathrm{s}$, Fig. S25 †) after removal of oxygen from the solvent. The observed rich dynamic processes implies that after photoexcitation the fluorescent molecule within the $\mathrm{G}_{1}-\mathrm{CB}[7]_{2}$ complex has sufficient structural freedom to relax to various low-energy excited states. On the other hand, the singular excited state dynamic observed for $\mathrm{G}_{2}-\mathrm{CB}[8]_{2}$ complex suggests a restricted or retarded structural change even in its excited state, which may affect both the radiative and non-radiative pathways.

2.8.3 Enhanced fluorescence efficiencies from constrained and discrete excited dimers. The rate constants corresponding to non-radiative $\left(k_{\mathrm{nr}}\right)$ and radiative $\left(k_{\mathrm{r}}\right)$ pathways are readily calculated from excited-state lifetime $\left(\tau_{\mathrm{s}}\right)$ and quantum yield $\left(\phi_{\mathrm{F}}\right)$ values. As shown in Table 1 , the formation of preorganized $\mathrm{G}_{2}-\mathrm{CB}[8]_{2}$ dimers always results in a reduced radiative rate constant $k_{\mathrm{r}}$ corresponding to a long-lived excimer-like excited state, which is smaller than the $k_{\mathrm{r}}$ values for the corresponding $\mathrm{G}_{1}-\mathrm{CB}[7]_{2}$ complexes. Contrary to typical excimers that lead to quenched emission, ${ }^{58} \quad \mathrm{G}_{2}-\mathrm{CB}[8]_{2}$ complexes maintain high fluorescence efficiencies on account of their substantial reduction in non-radiative rate constants $k_{\mathrm{nr}}$. This unique feature is attributed to a significant suppression of non-radiative deactivation through the formation of a preorganized dimer in $\mathrm{G}_{2}-\mathrm{CB}[8]_{2}$, which strongly restricts intracomplex motions as demonstrated above.

In addition to constrained complexation, the discrete nature of fluorophore dimers is also crucial to ensure high-efficiency fluorescence. ${ }^{60,61}$ The two $\mathrm{CB}[8]$ macrocycles that hold the fluorophore dimer together will mechanically block interactions from other dimers in aqueous solution, which effectively avoids the generation of dark excited states caused by arbitrary aggregation.

2.8.4 Comparison with other dimeric systems. An advantage of forming preorganized $\pi$-stacked ground-state dimers is that the excitation wavelength for the system is shifted towards the visible region (e.g. $469 \mathrm{~nm}$ for the $\mathrm{G}_{2}-\mathrm{CB}[8]_{2}$ complex of Ant910Me), which is crucial for non-destructive imaging of biological systems. Importantly, the formation of a preorganized $\pi$-stacked ground-state dimer is not necessarily the same as bringing together two fluorophores into spatial proximity. For instance, a red-shift in the absorption band was not observed in previous reports where two fluorophores have been covalently linked together in close proximity. ${ }^{2,48,57}$ The preorganization of $\pi$-stacked dimers of anthracene and its derivatives through non-covalent methods have been previously realized in rigid media containing small discrete cavities, such as crystalline lattices ${ }^{60-63}$ and supramolecular capsules, ${ }^{64}$ suggesting that the formation of a preformed $\pi$-stacked dimer requires strict spatial confinement in order to: (1) isolate each dimer as a discrete entity, (2) maintain a specific $\pi$-stacked configuration, and (3) restrict interplanar spacing between the two fluorophores.

The spontaneously assembled $\mathrm{G}_{2}-\mathrm{CB}[8]_{2}$ complex satisfies all three requirements and facilitates the formation of preorganized $\pi$-stacked dimers. The two fluorophores inside a $\mathrm{G}_{2}-\mathrm{CB}[8]_{2}$ complex form a discrete dimeric stack with a significant overlap of $\pi$ electrons and a restricted interplanar spacing defined by the $\mathrm{CB}[8]$ cavities. Steric hindrance from both $\mathrm{CB}[8]$ macrocycles facilitates "mechanical" separation between all dimers in aqueous solution ensuring pairwise fluorophores perform as a discrete entity. More importantly, the dimers are stabilized by $\mathrm{CB}[8]$ clamping and remain as such for a sufficiently long period of time. Finally, discrete preorganized dimers can be readily obtained through our strategy in aqueous solution at ambient temperatures, and therefore do not require formation of a specific crystal $^{60,61}$ or crystalline solvent at extremely low temperature. ${ }^{62,63}$ Moreover, owing our modular design, a variety of fluorophores are incorporated to give the corresponding $\pi$ - 
stacked dimers without any limitation on fluorophore size in direct contrast to other methods. ${ }^{64}$

\subsection{Controlling photophysics by clamping modules}

2.9.1 Suppression of radiative deactivation through nonparallel clamping. Complexation enhanced fluorescence is trivial for naphthyl-based guest molecules on account of their intrinsically high fluorescence efficiencies, whose quantum yield is almost unity even without complexation. An exception is Np27Me whose $\mathrm{G}_{2}-\mathrm{CB}[8]_{2}$ complex exhibits a quantum yield of 0.55 , which is about $40 \%$ less than its $\mathrm{G}_{1}-\mathrm{CB}[7]_{2}$ complex or in a non-complexed solution, and much smaller than the $\mathrm{G}_{2}-\mathrm{CB}[8]_{2}$ complexes of other naphthyl homologues (Table 1). A reduction in quantum yield is accompanied by a dramatic decrease in the radiative rate constant, which, in turn, results in the longest fluorescence lifetime observed for any species in this study of up to $36.8 \mathrm{~ns}$. A similar suppression in the radiative pathway is also observed for the $\mathrm{G}_{2}-\mathrm{CB}[8]_{2}$ complex of Ph13Me and the $\mathrm{G}_{2}-\mathrm{CB}[8]_{3}$ complex of Ph135Me, both of which exhibit a decreased quantum yield and an elongated lifetime. All three of these fluorescent molecules employ a non-parallel arrangement between their clamping modules, which suggests that non-parallel arrangements suppress the decay through radiative pathways. Clamping the dimer together in a non-parallel manner prevents any slippage of the two fluorophores along their extended axis and strongly restricts any intracomplex motions. As a consequence, the structural relaxation of the complex after photo-excitation towards a low-energy excited state is further retarded due to conformational rigidity amplified by non-parallel clamping. This restriction of motion is even more significant in triple-clamping cases, such as Ph135Me whose $k_{\mathrm{r}}$ and $\phi_{\mathrm{F}}$ values are reduced compared to Ph13Me.

Radiative decay is practically prohibited in the case of Ant14Me when it is complexed with $\mathrm{CB}[8]$, either by dual clamping or by triple clamping, exhibiting negligible quantum yield in either case (Table 1). Fluorescence quenching in the $\mathrm{G}_{2}-$ $\mathrm{CB}[8]_{3}$ complex of Ant14Me may be readily explained by triple clamping, however, it does not explain why complete quenching is also observed for its $\mathrm{G}_{2}-\mathrm{CB}[8]_{2}$ counterpart. One hypothesis is that the protruding anthracenyl moieties in one dimer may be long enough to interact with other protruding anthracenyl pairs located in another dimer, leading to some radiationless decay pathways that quickly deactivate the excited state. Interactions between protruding anthracenyl moieties is also supported by the diminished quantum yield observed for its $\mathrm{G}_{1}-\mathrm{CB}[7]_{2}$ complex compared to that of other anthracenyl homologues. Another possibility leading to radiationless decay may be a transition from a singlet to a triplet state through intersystem crossing, however, this requires substantial further study of the dynamics of Ant14Me complexes in their excited states.

2.9.2 H-H and H-T stacking of the fluorophore dimer. When the $\mathrm{G}_{2}-\mathrm{CB}[8]_{2}$ complex contains non-parallel clamping modules (e.g. Np27Me, Ph13Me, and Ph135Me), the way in which the two fluorophores are stacked with respect to one another is fixed. However, co-facial stacking of the two fluorophores may adopt either a head-to-head $(\mathrm{H}-\mathrm{H})$ or head-to-tail
(H-T) configuration when the clamping modules are parallel. This is not an issue for symmetric fluorophores such as Ant910Me and Ph14Me, as the $\mathrm{H}-\mathrm{H}$ and $\mathrm{H}-\mathrm{T}$ orientations are indistinguishable.

Interestingly, the $\mathrm{G}_{2}-\mathrm{CB}[8]_{2}$ complex of Ant15Me also adopts a single $\mathrm{H}-\mathrm{H}$ stacking configuration, as the spacing between its two off-line clamping modules is too large to allow for a feasible $\mathrm{H}-\mathrm{T}$ configuration (Scheme $\mathrm{S} 3 \dagger$ ). This specific stacking configuration is also revealed in the ${ }^{1} \mathrm{H}$ NMR spectrum of its $\mathrm{G}_{2}-\mathrm{CB}[8]_{2}$ complex, in which the protons of the 1,5-anthracenyl moieties exhibit sharp and well-resolved signals (Fig. S9†). In contrast, Np15Me with a smaller gap between its off-line clamping modules may allow for both $\mathrm{H}-\mathrm{H}$ and $\mathrm{H}-\mathrm{T}$ stacking configurations of the two fluorophores, which leads to a significant broadening of proton signals in the NMR of its $\mathrm{G}_{2}-\mathrm{CB}[8]_{2}$ complex. Moreover, all the proton signals are equally broadened suggesting a dynamic process that involves the entire complex, which very likely correlates to an interconversion between $\mathrm{H}-\mathrm{H}$ and $\mathrm{H}$-T stacking configurations with an exchanging rate on the intermediate NMR timescale (Fig. S7 $\dagger$ ). As a result, the aromatic fluorophores in the $\mathrm{G}_{2}-\mathrm{CB}[8]_{2}$ complexes Ant15Me and Np15Me both exhibit a substantial overlap of $\pi$-electrons in a less $J$ aggregate-like fashion, leading to smaller bathochromic shifts in their emission maxima (Fig. 5) compared to other fluorescent molecules. ${ }^{65}$ Considering their red-shift in absorption, the smaller bathochromic shifts in emission maxima may also correlate to an anti-Kasha behavior as mentioned above, which requires further investigation.

Although both $\mathrm{H}-\mathrm{H}$ and $\mathrm{H}-\mathrm{T}$ stacking should be feasible by Np14Me and Ant14Me, the NMR spectra of their $\mathrm{G}_{2}-\mathrm{CB}[8]_{2}$ complexes suggest a preference towards head-to-head stacking. The protons residing on the protruding ring exhibit an upfield shift due to shielding of the aromatic ring current, which is best explained by a head-to-head overlapping of the fluorophores. This further suggests that the $\pi-\pi$ interactions play a role in determining energy-favorable stacking configurations.

2.9.3 Substituents on the clamping modules. In addition to methyl (Me) groups in the para-position of the aryl clamping modules, other substituents including amino- $\left(\mathrm{NH}_{2}\right)$, methoxy(OMe), dimethylamino- $\left(\mathrm{NMe}_{2}\right)$, isopropyl- $\left(\mathrm{CMe}_{2}\right)$, and methylthio- (SMe) readily form monomeric and dimeric complexes with $\mathrm{CB}[7]$ and $\mathrm{CB}[8]$, respectively, in the same manner as the parent methyl compounds. As the aryl clamping modules are both bound inside the $\mathrm{CB}$ cavity for the $\mathrm{G}_{1}-\mathrm{CB}[7]_{2}$ and $\mathrm{G}_{2}-\mathrm{CB}[8]_{2}$ complexes, they exhibit similar diffusion coefficients regardless of the variation in para-substituents (Fig. 3b).

On the other hand, the photophysical properties of the dimeric stacked fluorophores are indeed affected by the size of the aryl substituents. As shown in Fig. 9, Np14H, a naphthyl fluorescent molecule without any substituent on its clamping module displays the same absorption and emission spectra as those of Np14Me. However, a significant difference of the emission maximum is observed for the $\mathrm{G}_{2}-\mathrm{CB}[8]_{2}$ complex of $\mathrm{Np14CMe}_{2}$. As both the absorption and emission spectra of $\mathrm{G}$ and $\mathrm{G}_{1}-\mathrm{CB}[7]_{2}$ of $\mathbf{N p 1 4 C M e} \mathbf{C}_{2}$ are similar to those of $\mathbf{N p 1 4 H}$ and Np14Me, this difference observed for the $\mathrm{G}_{2}-\mathrm{CB}[8]_{2}$ complex must stem from a certain variation in the stacking of the 

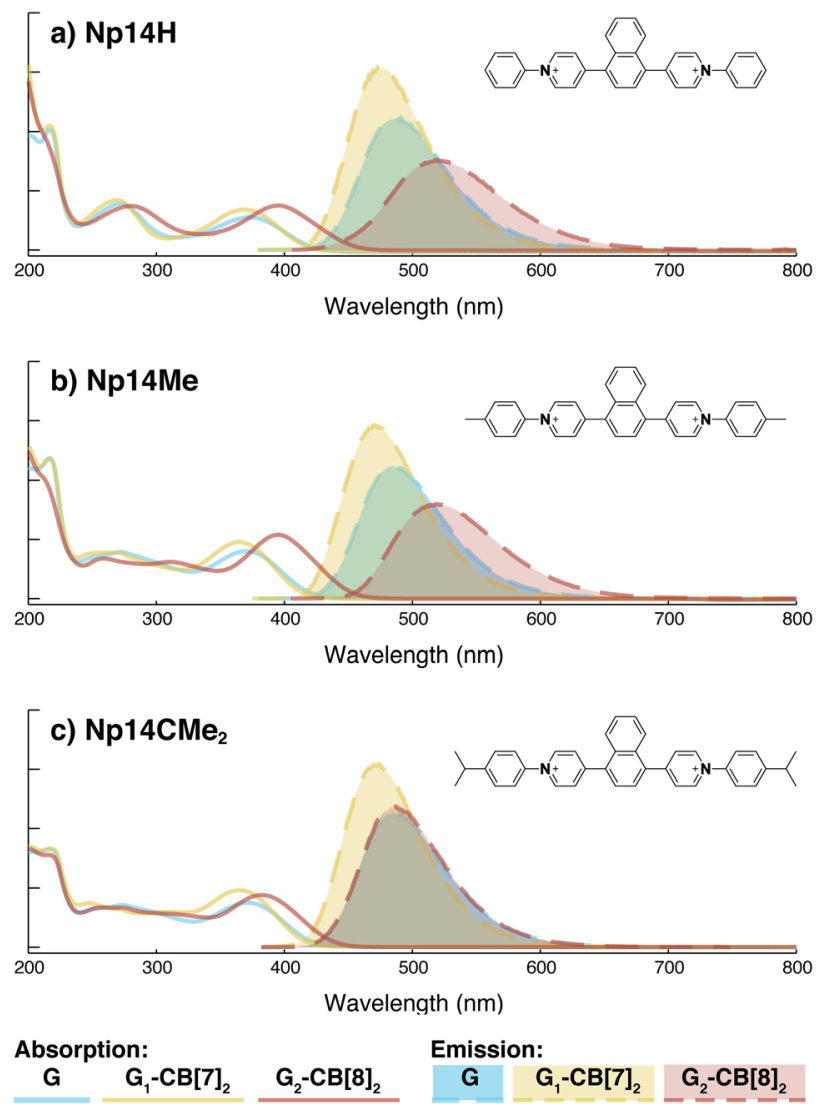

Fig. 9 Absorption (solid line) and emission (dash line with filling color) spectra of 1,4-naphthyl based fluorescent molecules (blue) with variation in substituents including (a) $\mathrm{Np} 14 \mathrm{H}$, (b) Np14Me, (c) $\mathrm{Np}_{14 C M e_{2}}$ along with their $\mathrm{G}_{1}-\mathrm{CB}[7]_{2}$ complexes (yellow) and $\mathrm{G}_{2}-\mathrm{CB}[8]_{2}$ complexes (red). Aqueous solution of each species are tested under a uniform guest concentration at $298 \mathrm{~K}$. The intensity is not normalized but scaled up by the same factor. The $\mathrm{Cl}^{-}$counterions are omitted for clarity.

naphthyl pair, which is very likely caused by a significant volume exclusion between neighboring isopropyl substituents. The resultant stacking configuration in $\mathrm{G}_{2}-\mathrm{CB}[8]_{2}$ of $\mathbf{N p 1 4 \mathbf { C M e } _ { 2 }}$ still leads to a red-shifted absorption band corresponding to $\pi$ electron delocalization in the preorganized dimer. It seems that the preorganized dimer (in this case) does not result in an effective formation of an excimer-like state, as the emission maximum is very similar to that in pristine solution without an obvious bathochromic shift. This observation thus offers an additional opportunity to tune the photophysical properties of stacked fluorophores by choosing appropriate substituents.

\section{Conclusions}

In summary, we have demonstrated a modular strategy to design a new class of fluorescent molecules that (i) generate discrete, dimeric stacked fluorophores in aqueous solution and (ii) are constrained by $\mathrm{CB}[8]-$-mediated multiple clamping. This modular design is surprisingly applicable and flexible and has been validated by testing nine different fluorophore cores ranging in size, shape, and geometric variation of their clamping modules. When complexed with $\mathrm{CB}[7]$, all fluorescent molecules are dispersed in aqueous solution as discrete monomers, exhibiting an impressively high fluorescence efficiency. On the other hand, complexation with $\mathrm{CB}[8]$ as $2: 2$ or $2: 3$ complexes leads to the immediate formation of discrete dimeric stacked fluorophores. Multiple $\mathrm{CB}[8]$ clamping results in stable, preorganized ground-state dimers, which can be readily photoexcited to excimer-like states, displaying significant bathochromic shifts in absorption and emission with elongated fluorescence lifetimes. Bathochromic shifts in the emission spectra can be readily tuned by controlling the stacking of fluorophores through specific variations in the clamping modules (through off-line alignment or altering substituents).

We demonstrate that intracomplex motion in the preorganized dimers is significantly restricted, which suppresses both radiative and non-radiative deactivation, resulting in a substantially high quantum yield (up to 1) despite formation of excimer-like states. Some complexes are further restricted through non-parallel or triple clamping, which slows down radiative relaxation to an even greater extent, leading to elongated excited-state lifetimes up to $37 \mathrm{~ns}$ in aqueous solution. Moreover, complexes stabilized by multiple non-parallel clamping exhibit self-sorting in the presence of excess $\mathrm{CB}[8]$, which facilitates the design and fabrication of hierarchical functional structures.

While only arylpyridinium moieties have been employed as the clamping module in this study, current investigations suggest other chemical motifs with rigid structure exhibit the same clamping feature. The high rigidity ensures intrinsically low conformational entropy change during complexation, thus facilitating the formation of a long-lived, multicomponent complex in aqueous solution.

From a fundamental point of view, this study offers a model system with explicitly stable dimeric structures and tuneable features that can be utilized as a platform to study various intermolecular processes including excimer formation, charge transfer, exciton coupling, and singlet fission. Moreover, such a modular molecular design towards quadrupolar fluorescent molecules may provide a feasible toolbox in pursuit of distinct features such as large two-photon cross-sections ${ }^{6,67}$ and nonKasha behavior. ${ }^{68}$ On the practical side, $\mathrm{CB}[7]-$ and $\mathrm{CB}[8]-$ mediated fluorescent complexes developed here are promising candidates for various (biological) imaging applications on account of their emergent photophysical properties such as long lifetimes, high emission brightness, and red-shifted excitation bands.

\section{Conflicts of interest}

There are no conflicts to declare.

\section{Acknowledgements}

This work was supported by Leverhulme Trust (project: "Natural material innovation for sustainable living"), the Marie Curie FP7 SASSYPOL ITN (607602) programme, EPSRC Programme 
Grant (NOtCH, EP/L027151/1), and ERC-2016 Consolidator Grant (CAM-RIG, 726470) (G. W., M. O., O. A. S.), the U. S. Department of Energy, Office of Science, Office of Basic Energy Sciences, under Award DE-FG02-99ER14999 (Y. J. B., M. R. W.), EPSRC (EP/M508007/1) (D. A. G.), and EPSRC (EP/R013012/1, $\mathrm{EP} / \mathrm{L027151/1}$ and EP/N020669/1), BBSRC (BB/N007700/1), ERC (project 757850 BioNet) (I. S., E. R.). The authors thank Dr Peter Grice and Mr Duncan Howe for their help on NMR analysis, and thank Prof Luisa De Cola, Dr Bruno Frka-Petesic, Dr Stefan Mommer, and Wiebke Schnettger for their suggestion.

\section{References}

1 M. A. Slifkin, Nature, 1963, 200, 766-767.

2 Y. Wu, J. Zhou, B. T. Phelan, C. M. Mauck, J. F. Stoddart, R. M. Young and M. R. Wasielewski, J. Am. Chem. Soc., 2017, 139, 14265-14276.

3 J. Hoche, H.-C. Schmitt, A. Humeniuk, I. Fischer, R. Mitrić and M. I. S. Röhr, Phys. Chem. Chem. Phys., 2017, 19, 25002-25015.

4 H. Tamura, J. Phys. Chem. A, 2016, 120, 9341-9347.

5 C. Kaufmann, D. Bialas, M. Stolte and F. Würthner, J. Am. Chem. Soc., 2018, 140, 9986-9995.

6 C. E. Miller, M. R. Wasielewski and G. C. Schatz, J. Phys. Chem. C, 2017, 121, 10345-10350.

7 Y. J. Bae, G. Kang, C. D. Malliakas, J. N. Nelson, J. Zhou, R. M. Young, Y.-L. Wu, R. P. Van Duyne, G. C. Schatz and M. R. Wasielewski, J. Am. Chem. Soc., 2018, 140, 1514015144.

8 A. Rao and R. H. Friend, Nat. Rev. Mater., 2017, 2, 17063.

9 Y. Hong, J. W. Y. Lam and B. Z. Tang, Chem. Soc. Rev., 2011, 40, 5361-5388.

10 F. Würthner, T. E. Kaiser and C. R. Saha-Möller, Angew. Chem., Int. Ed., 2011, 50, 3376-3410.

11 B. Heyne, Photochem. Photobiol. Sci., 2016, 15, 1103-1114.

12 R. N. Dsouza, U. Pischel and W. M. Nau, Chem. Rev., 2011, 111, 7941-7980.

13 S. Anderson and H. L. Anderson, Angew. Chem., Int. Ed., 1996, 35, 1956-1959.

14 J. C. Barnes, M. Juríček, N. L. Strutt, M. Frasconi, S. Sampath, M. A. Giesener, P. L. McGrier, C. J. Bruns, C. L. Stern, A. A. Sarjeant and J. F. Stoddart, J. Am. Chem. Soc., 2012, 135, 183-192.

15 E. J. Dale, N. A. Vermeulen, M. Juricek, J. C. Barnes, R. M. Young, M. R. Wasielewski and J. F. Stoddart, Acc. Chem. Res., 2016, 49, 262-273.

16 L. Yang, P. Langer, E. S. Davies, M. Baldoni, K. Wickham, N. A. Besley, E. Besley and N. R. Champness, Chem. Sci., 2019, 10, 3723-3732.

17 S. J. Barrow, S. Kasera, M. J. Rowland, J. del Barrio and O. A. Scherman, Chem. Rev., 2015, 115, 12320-12406.

18 K. I. Assaf and W. M. Nau, Chem. Soc. Rev., 2015, 44, 394-418.

19 A. L. Koner and W. M. Nau, Supramol. Chem., 2007, 19, 5566.

20 J. Mohanty and W. M. Nau, Angew. Chem., Int. Ed., 2005, 44, 3750-3754.
21 M. Megyesi, L. Biczók and I. Jablonkai, J. Phys. Chem. C, 2008, 112, 3410-3416.

22 M. Freitag, L. Gundlach, P. Piotrowiak and E. Galoppini, J. Am. Chem. Soc., 2012, 134, 3358-3366.

23 K. Liu, Y. Yao, Y. Kang, Y. Liu, Y. Han, Y. Wang, Z. Li and X. Zhang, Sci. Rep., 2013, 3, 2372.

24 H.-J. Kim, J. Heo, W. S. Jeon, E. Lee, J. Kim, S. Sakamoto, K. Yamaguchi and K. Kim, Angew. Chem., Int. Ed., 2001, 40, 1526-1529.

25 L. M. Heitmann, A. B. Taylor, P. J. Hart and A. R. Urbach, J. Am. Chem. Soc., 2006, 128, 12574-12581.

26 S. Senler, W. Li, M. H. Tootoonchi, S. Yi and A. E. Kaifer, Supramol. Chem., 2014, 26, 677-683.

27 P. Montes-Navajas and H. Garcia, J. Phys. Chem. C, 2010, 114, 2034-2038.

28 G. Wu, M. Olesińska, Y. Wu, D. Matak-Vinkovic and O. A. Scherman, J. Am. Chem. Soc., 2017, 139, 3202-3208.

29 H. Yin, Q. Cheng, R. Rosas, S. Viel, V. Monnier, L. Charles, D. Siri, D. Gigmes, O. Ouari, R. Wang, et al., Chem.-Eur. J., 2019, 25, 12552-12559.

30 S. Chakrabarti and L. Isaacs, Supramol. Chem., 2008, 20, 191199.

31 J. Mohanty, S. Dutta Choudhury, H. P. Upadhyaya, A. C. Bhasikuttan and H. Pal, Chem.-Eur. J., 2009, 15, 5215-5219.

32 J. Mohanty, N. Thakur, S. Dutta Choudhury, N. Barooah, H. Pal and A. C. Bhasikuttan, J. Phys. Chem. B, 2012, 116, 130-135.

33 B. Yang, S.-B. Yu, H. Wang, D.-W. Zhang and Z.-T. Li, Chem.Asian J., 2018, 13, 1312-1317.

34 K. Kotturi and E. Masson, Chem.-Eur. J., 2018, 24, 86708678.

35 S. Schoder, H. V. Schröder, L. Cera, R. Puttreddy, A. Güttler, U. Resch-Genger, K. Rissanen and C. A. Schalley, Chem.-Eur. J., 2019, 25, 3257-3261.

36 T. K. Ronson, W. Meng and J. R. Nitschke, J. Am. Chem. Soc., 2017, 139, 9698-9707.

37 D. Bongard, M. Möller, S. N. Rao, D. Corr and L. Walder, Helv. Chim. Acta, 2005, 88, 3200-3209.

38 M. Olesińska, G. Wu, S. Gómez-Coca, D. Antoón-Garciía, I. Szabó, E. Rosta and O. A. Scherman, Chem. Sci., 2019, 10, 8806-8811.

39 G. Wu, I. Szabó, E. Rosta and O. A. Scherman, Chem. Commun., 2019, 55, 13227-13230.

40 W. L. Mock and N. Y. Shih, J. Org. Chem., 1986, 51, 44404446.

41 G. Wu, D. E. Clarke, C. Wu and O. A. Scherman, Org. Biomol. Chem., 2019, 17, 3514-3520.

42 Y. H. Ko, K. Kim, E. Kim and K. Kim, Supramol. Chem., 2007, 19, 287-293.

43 S. Kohmoto, T. Chuko, S. Hisamatsu, Y. Okuda, H. Masu, M. Takahashi and K. Kishikawa, Cryst. Growth Des., 2015, 15, 2723-2731.

44 H.-J. Kim, D. R. Whang, J. Gierschner and S. Y. Park, Angew. Chem., Int. Ed., 2016, 55, 15915-15919.

45 X.-L. Ni, S. Chen, Y. Yang and Z. Tao, J. Am. Chem. Soc., 2016, 138, 6177-6183. 
46 R. Ye, Q. Cui, C. Yao, R. Liu and L. Li, Phys. Chem. Chem. Phys., 2017, 19, 31306-31315.

47 B. Zhang, Y. Dong, J. Li, Y. Yu, C. Li and L. Cao, Chin. J. Chem., 2019, 37, 269-275.

48 H. Osaki, C.-M. Chou, M. Taki, K. Welke, D. Yokogawa, S. Irle, Y. Sato, T. Higashiyama, S. Saito, A. Fukazawa and S. Yamaguchi, Angew. Chem., Int. Ed., 2016, 55, 7131-7135.

49 J. V. Morris, M. A. Mahaney and J. R. Huber, J. Phys. Chem., 1976, 80, 969-974.

50 G. M. Blackburn, G. Lockwood and V. Solan, J. Chem. Soc., Perkin Trans. 2, 1976, 1452-1456.

51 V. A. Kharlanov, M. I. Knyazhansky, N. I. Makarova and V. A. Lokshin, J. Photochem. Photobiol., A, 1993, 70, 223-227.

52 F. C. Spano and C. Silva, Annu. Rev. Phys. Chem., 2014, 65, 477-500.

53 A. Mulder, J. Huskens and D. N. Reinhoudt, Org. Biomol. Chem., 2004, 2, 3409-3424.

54 J. K. M. Sanders and K. Brian, Modern NMR Spectroscopy, a Guide for Chemists, Oxford University. Oxford, GB, 1993.

55 R. Joseph and E. Masson, Eur. J. Org. Chem., 2014, 2014, 105110.

56 M. Jin, T. S. Chung, T. Seki, H. Ito and M. A. Garcia-Garibay, J. Am. Chem. Soc., 2017, 139, 18115-18121.

57 S. Akine, T. Onuma and T. Nabeshima, New J. Chem., 2018, 42, 9369-9372.
58 J. B. Birks, Photophysics of Aromatic Molecules, 1970.

59 S. K. Pal, J. Peon, B. Bagchi and A. H. Zewail, J. Phys. Chem. B, 2002, 106, 12376-12395.

60 H. Liu, L. Yao, B. Li, X. Chen, Y. Gao, S. Zhang, W. Li, P. Lu, B. Yang and Y. Ma, Chem. Commun., 2016, 52, 7356-7359.

61 Y. Shen, H. Liu, S. Zhang, Y. Gao, B. Li, Y. Yan, Y. Hu, L. Zhao and B. Yang, J. Mater. Chem. C, 2017, 5, 10061-10067.

62 E. A. Chandross, J. Ferguson and E. G. McRae, J. Chem. Phys., 1966, 45, 3546-3553.

63 E. A. Chandross and J. Ferguson, J. Chem. Phys., 1966, 45, 3554-3564.

64 L. S. Kaanumalle, C. L. D. Gibb, B. C. Gibb and V. Ramamurthy, J. Am. Chem. Soc., 2005, 127, 3674-3675.

65 Y. Gao, H. Liu, S. Zhang, Q. Gu, Y. Shen, Y. Ge and B. Yang, Phys. Chem. Chem. Phys., 2018, 20, 12129-12137.

66 M. Albota, D. Beljonne, J.-L. Brédas, J. E. Ehrlich, J.-Y. Fu, A. A. Heikal, S. E. Hess, T. Kogej, M. D. Levin, S. R. Marder, D. McCord-Maughon, J. W. Perry, H. Röckel, M. Rumi, G. Subramaniam, W. W. Webb, X.-L. Wu and C. Xu, Science, 1998, 281, 1653-1656.

67 G. D'Avino, F. Terenziani and A. Painelli, J. Phys. Chem. B, 2006, 110, 25590-25592.

68 C. Zheng, C. Zhong, C. J. Collison and F. C. Spano, J. Phys. Chem. C, 2019, 123, 3203-3215. 